\title{
A next generation approach to species delimitation reveals the role of hybridization in a cryptic species complex of corals
}

Andrea M. Quattrini ${ }^{1 *}$ D, Tiana Wu' ${ }^{1}$ Keryea Soong ${ }^{2}$, Ming-Shiou Jeng ${ }^{3}$, Yehuda Benayahu ${ }^{4}$ and Catherine S. McFadden ${ }^{1}$

\begin{abstract}
Background: Our ability to investigate processes shaping the evolutionary diversification of corals (Cnidaria: Anthozoa) is limited by a lack of understanding of species boundaries. Discerning species of corals has been challenging due to a multitude of factors, including homoplasious and plastic morphological characters and the use of molecular markers that are either not informative or have not completely sorted. Hybridization can also blur species boundaries by leading to incongruence between morphology and genetics. We used traditional DNA barcoding and restriction-site associated DNA sequencing combined with coalescence-based and allele-frequency methods to elucidate species boundaries and simultaneously examine the potential role of hybridization in a speciose genus of octocoral, Sinularia.

Results: Species delimitations using two widely used DNA barcode markers, mtMutS and $28 \mathrm{~S}$ rDNA, were incongruent with one another and with the morphospecies identifications. When mtMutS and 285 were concatenated, a $0.3 \%$ genetic distance threshold delimited the majority of morphospecies. In contrast, 12 of the 15 examined morphospecies formed well-supported monophyletic clades in both concatenated RAXML phylogenies and SNAPP species trees of $>6000$ RADSeq loci. DAPC and Structure analyses also supported morphospecies assignments, but indicated the potential for two additional cryptic species. Three morphologically distinct species pairs could not, however, be distinguished genetically. ABBA-BABA tests demonstrated significant admixture between some of those species, suggesting that hybridization may confound species delimitation in Sinularia.
\end{abstract}

Conclusions: A genomic approach can help to guide species delimitation while simultaneously elucidating the processes generating coral diversity. Results support the hypothesis that hybridization is an important mechanism in the evolution of Anthozoa, including octocorals, and future research should examine the contribution of this mechanism in generating diversity across the coral tree of life.

Keywords: Hybridization, RADSeq, Phylogenetics, Coral reefs, Taxonomy, Anthozoa, Octocorallia

\footnotetext{
* Correspondence: aquattrini@g.hmc.edu

${ }^{1}$ Biology Department, Harvey Mudd College, 1250 N. Dartmouth Ave,

Claremont, CA 91711, USA

Full list of author information is available at the end of the article
}

(c) The Author(s). 2019 Open Access This article is distributed under the terms of the Creative Commons Attribution 4.0 International License (http://creativecommons.org/licenses/by/4.0/), which permits unrestricted use, distribution, and reproduction in any medium, provided you give appropriate credit to the original author(s) and the source, provide a link to the Creative Commons license, and indicate if changes were made. The Creative Commons Public Domain Dedication waiver (http://creativecommons.org/publicdomain/zero/1.0/) applies to the data made available in this article, unless otherwise stated. 


\section{Background}

The ability to delimit species is fundamental to the accurate assessment of biodiversity and biogeography, information that is essential for studying community ecology as well as for implementing conservation policies. Yet this task is not trivial, as species are often difficult to discriminate for a multitude of reasons. Morphological traits have traditionally been used in classical taxonomy; however, use of characters that might not be diagnostic or are homoplasious can confound the interpretation of species boundaries. Cryptic species, particularly those that occur in sympatry, and species that have arisen via hybridization and introgression are often challenging to discriminate without genetic, ecological or behavioral data. DNA barcoding of mitochondrial genes has proven useful in many species groups [46, 47], but incomplete lineage sorting and past hybridization events complicate species delimitation based on mitochondrial data alone, particularly in recently diverged taxa $[49,53,73]$. In addition, mitochondrial markers reflect the history of maternal lineages, which are often incongruent with the species' history $[18,89]$. The increased resolution of genomic data can potentially disentangle some of these issues, facilitating species delimitation while simultaneously furthering our understanding of processes that generate biodiversity [103]. Moreover, such an approach may also provide a better evaluation of morphological traits and insights into their congruence with genetic data.

In sessile marine invertebrates, such as corals, congeners often occur in sympatry and occupy similar ecological niches and reef zones. These ecological characteristics combined with reproductive modes may lead to increased rates of hybridization among close relatives. Broadcastspawning species that occur in sympatry often participate in synchronous, mass-spawning reproduction events [2, $44,55,88]$. Unless there are prezygotic mechanisms to reproductive isolation, such as gametic incompatibility or asynchronous spawning times, there may be numerous opportunities for hybridization to occur [109, 113, 114]. In fact, laboratory crossings of sympatric congeners have produced viable hybrid offspring in several species [101, 113]. Hybridization followed by reticulate evolution has been suggested to be an important mechanism generating the species diversity observed in some groups of corals $[17,20,39,45,69,74,87,108,109,114]$. Vollmer and Palumbi [112], however, suggested that hybridization could yield distinct, new morphotypes that may be reproductively inviable or subject to hybrid breakdown. It is clear that further investigation is needed to determine the potential contributions of hybridization to speciation and morphological innovation in corals.

One particularly speciose group of octocorals (Cnidaria: Anthozoa: Octocorallia) is the genus Sinularia May, 1898. This zooxanthellate genus includes approximately 175 valid species [115], 47 of them described just in the last 25 years. They are diverse and abundant throughout the Indo-Pacific, and biodiversity surveys of shallow-water coral reef communities typically report more than 15 co-occurring species of Sinularia, with as many as 38 species recorded at some locations $[67,75,76]$. Sinularia species are typically most abundant on reef flats and shallow slopes, where single- or multi-species assemblages may dominate the reef substrate $[4,5,21,31,105]$. Sinularia can also play an active role in reef biogenesis through deposition of spiculite formed by the cementation of layers of calcitic sclerites [52, 98]. In addition, many Sinularia species produce secondary metabolites used for allelopathy and predator deterrence [99, 100, 106, 116], making the genus a rich and diverse source of bioactive natural products (e.g., Blunt et al., [9]).

Because of the dominance and importance of Sinularia species across a wide depth gradient [97] as well as their susceptibility to bleaching-induced mortality [12, $32,41,68]$, it is of great interest to better understand their ecology and function on the reefs. Ecological studies, however, are often hampered by the uncertainty of species identifications [70]. Classical taxonomy of Sinularia species is based primarily on morphological features of the colony and the shape and dimension of sclerites (microscopic calcitic skeletal elements) found in different parts of the colony [33, 70, 111]. Separation of species using these characters can be subjective, as the complex morphologies of both colonies and sclerites are rarely quantified $[1,14]$. There is also a potential contribution of environmental plasticity to the morphological variation observed, as has been documented in other octocorals [59, 92, 93].

The application of molecular systematic and DNA barcoding approaches to the study of species boundaries in Sinularia have been only partially successful [70]. While molecular approaches have revealed that some well-known morphospecies comprise cryptic species complexes [77, 78 ], it is also the case that numerous morphologically distinct Sinularia species share identical haplotypes at barcoding loci $[8,70,72]$. Because mitochondrial genes evolve slowly in Anthozoa [50, 96], these markers often simply lack the resolution to distinguish recently diverged species $[71,72]$. As a result, it is often not possible to conclude with certainty whether morphologically distinct individuals that share identical DNA barcodes represent different octocoral species or morphological variants of a single species. In addition, the reported ability of some species of Sinularia to hybridize in the laboratory [101], raises the possibility that naturally occurring hybridization events could contribute to the observed morphological diversity of this genus, as has been suggested for stony corals [87]. The true identity of some Sinularia species remains uncertain, and our ability to explore the evolutionary processes 
leading to diversification of this hyperdiverse lineage are limited by a lack of understanding of species boundaries. In fact, we have a limited understanding of species boundaries for numerous groups of recently diverged corals (e.g., $[53,73,107])$.

Octocoral biodiversity surveys conducted recently at Dongsha Atoll, South China Sea, Taiwan, recorded 27 nominal morphospecies of Sinularia inhabiting the reef slope down to a depth of $20 \mathrm{~m}$ [8], most of them belonging to the speciose clades " 4 " and " 5 " [70]. These two clades include several subclades each characterized by a different suite of morphological characters whose diagnosis is quite confusing [70]. While most of these morphospecies could be distinguished using a character-based mitochondrial gene barcode ( $m t M u t S$ ), five distinct morphospecies in clade $5 \mathrm{C}$ shared identical haplotypes, and several morphospecies in both clades were represented by more than one haplotype [8]. These morphospecies exemplify a problem common to many corals and raise the following questions: (1) do the observed morphological differences reflect boundaries between species whose mitochondrial haplotypes have not yet diverged or coalesced, or do these differences reflect intraspecific variation? and (2) might the sharing of mitochondrial haplotypes among distinct morphotypes reflect ongoing or past hybridization events?

To further explore these questions and to elucidate species boundaries in the Sinularia that co-occur on Dongsha Atoll, we have (1) sequenced an additional, nuclear marker (28S rDNA) that has been shown to be comparable to $m t M u t S$ as a species-specific barcode for Sinularia and other octocoral taxa [72]; and (2) sequenced restriction-site associated DNA (RADseq) to identify SNPs for multilocus species delimitation analyses using allele-frequency and coalescence-based approaches. Expanding upon a recent study of co-occurring Sinularia species at Dongsha Atoll [8], we validate morphospecies identifications using a genomic approach, and provide insight into the possible role of hybridization in the evolution of the genus.

\section{Results}

\section{Species delimitation using DNA barcodes}

Neither the mtMutS (735 bp) nor the 28S rDNA (764 bp) barcoding marker delimited all morphospecies of Sinularia (Table 1) when considered separately (Additional file 1: Figure S1, Additional file 4). Phylogenetic relationships among morphospecies were poorly resolved with low support values and few reciprocally monophyletic groups, especially in clade 5C (Additional file 1: Figure S1). Based on a $0.3 \%$ genetic distance threshold, the mtMutS barcode identified six molecular operational taxonomic units (MOTUs) among the four morphospecies belonging to clade 4, splitting $S$. tumulosa and S. ceramensis into two MOTUs each (Additional file 1: Figure S1). In contrast, $28 S$ rDNA (0.3\% threshold) delimited only four MOTUs within clade 4, each of them congruent with morphospecies identifications. The only exception was $S$. verruca (R41341) from Palau, included as a taxonomic reference, whose $m t M u t S$ and $28 S$ sequences were identical to those of $S$. tumulosa. When the two barcode markers were concatenated, application of a $0.3 \%$ genetic

Table 1 Morphospecies of Sinularia included in RADseq analysis

\begin{tabular}{|c|c|c|c|c|c|}
\hline Species & Authority & Clade & $\mathrm{N}$ & \#Sites & Depth (m) \\
\hline S. abrupta & Tixier-Durivault, 1970 & $5 C$ & 3 & 2 & $8-13$ \\
\hline S. acuta & Manuputty \& Ofwegen, 2007 & $5 C$ & 11 & 6 & $4-14$ \\
\hline S. ceramensis & Verseveldt, 1977 & $4 \mathrm{D}$ & 6 & 5 & $3-13$ \\
\hline S. daii ${ }^{\mathrm{a}}$ & Benayahu \& Ofwegen, 2011 & $5 C$ & 1 & - & --- \\
\hline S. densa & (Whitelegge, 1897) & $5 C$ & 6 & 4 & $3-14$ \\
\hline S. exilis & Tixier-Durivault, 1970 & $5 C$ & 2 & 2 & $5-14$ \\
\hline S. humilis & Ofwegen, 2008 & $4 \mathrm{~B}$ & 4 & 3 & $3-21$ \\
\hline S. lochmodes & Kolonko, 1926 & $5 C$ & 8 & 6 & $4-14$ \\
\hline S. maxima & Verseveldt, 1971 & $5 C$ & 3 & 2 & $3-17$ \\
\hline S. pavida & Tixier-Durivault, 1970 & $4 \mathrm{D}$ & 2 & 2 & $4-5$ \\
\hline S. penghuensis & Ofwegen 2012 & $5 C$ & 8 & 3 & $3-21$ \\
\hline S. slieringsi & Ofwegen \& Vennam, 1994 & $5 C$ & 17 & 6 & $3-21$ \\
\hline S. tumulosa & Ofwegen, 2008 & $4 \mathrm{D}$ & 12 & 8 & $3-17$ \\
\hline S. verruca ${ }^{a}$ & Ofwegen, 2008 & $4 \mathrm{D}$ & 1 & - & --- \\
\hline S. wanannensis & Ofwegen 2012 & $5 C$ & 9 & 5 & $5-21$ \\
\hline
\end{tabular}

Clade corresponds to designations in McFadden et al. [70]. N: number of specimens sequenced. \#Sites: number of different dive sites at Dongsha Atoll at which a species was collected

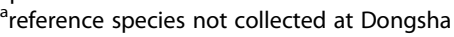


distance threshold delimited five species in clade 4: $S$. humilis, S. pavida and S. ceramensis were delimited clearly, but individuals of $S$. tumulosa were split among two putative species, one of which included $S$. verruca (Fig. 1a).

Among the clade $5 \mathrm{C}$ morphospecies, $m t M u t S$ delineated eight MOTUs (Additional file 1: Figure S1). With just two exceptions (D442, Z34695), mtMutS differentiated S. maxima, S. wanannensis, S. lochmodes and S. densa from all

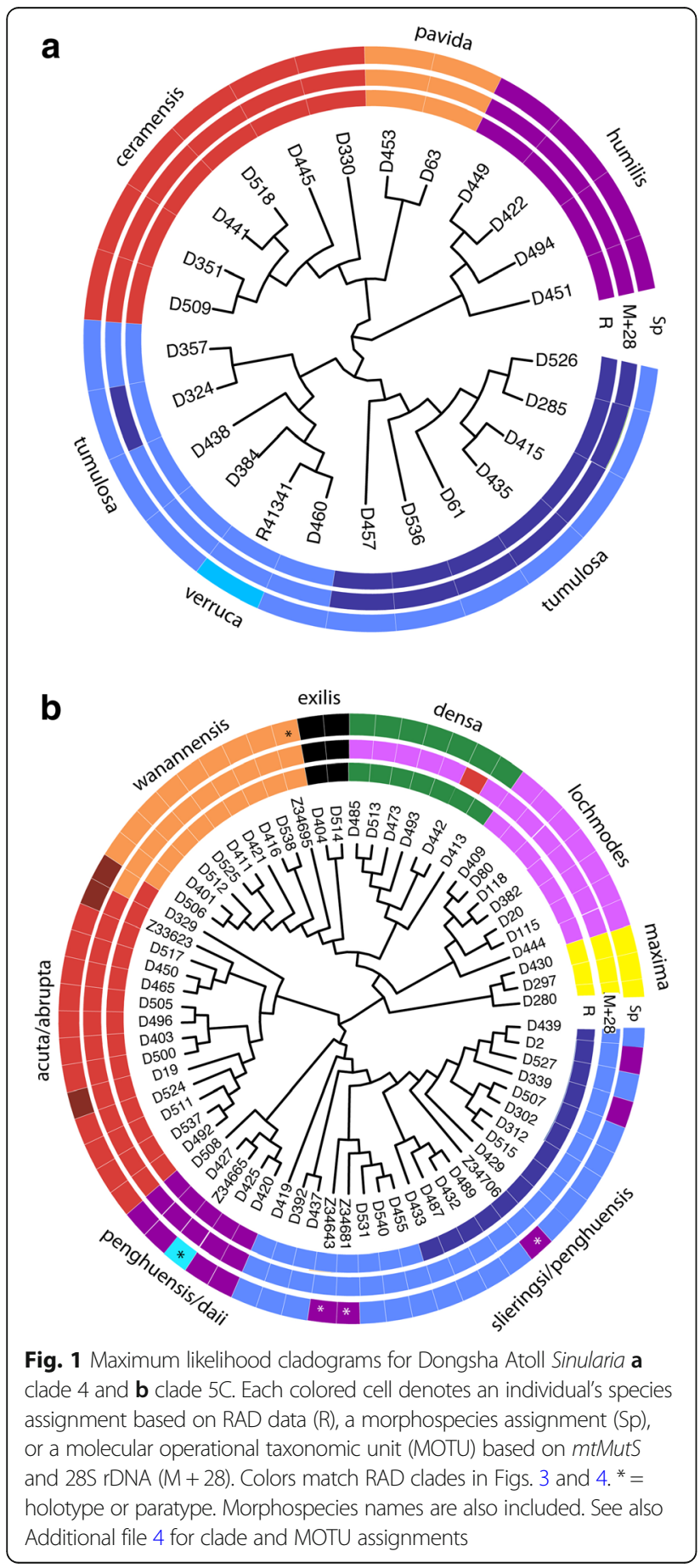

other morphospecies (Additional file 1: Figure S1). A majority of the colonies identified as $S$. penghuensis and $S$. slieringsi, both individuals of $S$. exilis, and seven of eleven S. acuta, however, belonged to a single MOTU, while the remaining four individuals of $S$. acuta were assigned to a separate MOTU. Two individuals with divergent haplotypes (S. penghuensis D002 and S. slieringsi D439) were each assigned to unique MOTUs. In contrast to $m t M u t S$, $28 S$ delineated only four MOTUs within clade 5C (Additional file 1: Figure S1). S. penghuensis and S. slieringsi, which were not separated using $m t M u t S$, were separated into two distinct MOTUs; four of the S. penghuensis colonies belonged to a MOTU that was well separated from all others and also included $S$. daii, while the other fiveincluding the holotype (Z34706) and both paratypesshared identical genotypes with $S$. slieringsi. A third MOTU included S. acuta, S. densa and S. lochmodes along with S. abrupta and one $S$. exilis. The fourth MOTU included all individuals of $S$. maxima and $S$. wanannensis plus the second $S$. exilis.

When mtMutS and $28 S$ were concatenated, a $0.3 \%$ genetic distance threshold delimited seven MOTUs in clade $5 \mathrm{C}$, five of them largely congruent with morphospecies definitions (S. maxima, S. exilis, S. acuta, S. wanannensis and S. slieringsi) (Fig. 1b; Additional file 1: Figure S2). S. penghuensis was divided among two MOTUs: five individuals (including all three type specimens) belonged to the same MOTU as S. slieringsi, while the other four were placed in a MOTU with $S$. daii. S. lochmodes and all but one individual of $S$. densa were lumped together in a seventh MOTU. Several individuals did not fall into a MOTU with others belonging to the same morphospecies: two individuals of $S$. abrupta (D119, Z33623) and one S. densa (D442) were not distinguished from $S$. acuta, while the third $S$. abrupta (D329) was lumped with $S$. wanannensis (Fig. 1b). Two of the individuals of S. abrupta showed incongruence between mtMutS and $28 S$ genotypes: D329 had a mtMutS sequence matching $S$. wanannensis but a $28 S$ sequence matching $S$. acuta, while Z33623 had a $m t M u t S$ sequence matching $S$. acuta but its $28 S$ matched $S$. densa. In contrast, D019 was identical to $S$. acuta at both loci. The holotype of $S$. wanannensis from Penghu (Z34695) also had incongruent barcode genotypes; its $28 S$ sequence was consistent with other individuals of that species, but it shared a $m t M u t S$ haplotype with S. penghuensis and S. slieringsi (Additional file 1: Figure S1).

\section{RADSeq data statistics}

A total of 289,373,374 reads were obtained for 95 Sinularia samples. After trimming in both Stacks and pyRAD, $86 \%$ of reads were retained $(247,873,622)$. The mean number of reads per individual was 2,609,196 \pm 
627,314 . For each clade, the number of loci and the number of SNPs obtained increased considerably when the number of shared heterozygous sites $(p)$ was increased and both the clustering threshold $(c)$ and individual occupancy per locus $(m)$ were decreased (Table 2). Notably, a substantial increase in both the number of loci and SNPs obtained occurred when $p$ was set to 0.25 at a clustering threshold $(c)$ of 0.85 . The number of loci obtained ranged from 73 to 28,179 for clade 4 and 115 to 23,946 for clade 5C depending upon parameters used in pyRAD analyses (Table 2). The number of variable SNPs obtained ranged from 382 to 251,615 for clade 4 and 885 to 329,837 for clade $5 \mathrm{C}$ (Table 2).

\section{Phylogenetic inference and species delimitation}

Similar to the combined analysis of concatenated $m t M u t S$ and $28 S$ rDNA barcodes (Fig. 1, Additional file 1: Figures S1 and S2), a majority of the identified morphospecies formed well-supported monophyletic clades in both clade 4 and $5 \mathrm{C}$ phylogenies constructed with the $c 0.85, p 0.25$, and $m 0.75$ RADSeq datasets (clade 4: 6,343 loci, clade 5C: 8,060 loci; Figs. 2, 3). The maximum clade credibility species trees produced from SNP data in the SNAPP analyses were largely congruent with the RAxML trees generated from concatenated data (Figs. 2, 3, 4). However, in clade 4, S. pavida and S. ceramensis were reciprocally monophyletic in the ML tree (Fig. 2) but not in the maximum clade credibility SNAPP species tree (Fig. 4a), although this relationship was evident in $30 \%$ of the alternative SNAPP tree topologies (in red, Fig. 4a). In clade 5C, S. acuta was sister to S. penghuensis and S. slieringsi in the ML tree (Fig. 3), but not in the maximum clade credibility species tree (Fig. 4b), although this relationship was evident in 25\% of the alternative SNAPP tree topologies (in red and green, Fig. 4b). For clade 4, 35\% of the SNAPP topologies obtained were alternative to the maximum clade tree, and for clade 5C 37\% of trees had different topologies compared to the maximum clade credibility tree (Fig. 4).

\section{Clade 4}

DAPC and Structure analyses supported the currently defined morphospecies in Sinularia clade 4 (S. ceramensis, S. humilis, S. pavida, and S. tumulosa), and agreed with the four MOTUs identified by the $28 S$ rDNA barcoding marker (Additional file 1: Figure S1). Consistent with the barcoding results, $S$. verruca was genetically indistinguishable from $S$. tumulosa. The optimal number of $K$ clusters suggested by the DAPC analyses was four $(\mathrm{BIC}=120.4$, Additional file 1 : Figure S3), and the DAPC plot revealed no overlap among these four distinct clusters (Fig. 5a). Further support for group assignment can be seen in the assignment plots, as all individuals were successfully re-assigned into their respective clusters (Additional file 1: Figure S4). In addition, the Distruct plot clearly illustrated little to no admixture among these four species (Fig. 2). Upon further Structure analysis, little to no admixture was also revealed between two sub-clades of $S$. tumulosa (Fig. 2), suggesting that $S$. tumulosa might consist of two species. Other methods also

Table 2 Dongsha Atoll Sinularia. Loci and SNP summary statistics of pyRAD simulations at two different clustering thresholds (c), three different levels of taxon occupancy per locus $(m)$, and two different levels of shared polymorphic sites ( $p$ ). Minimum and maximum loci obtained for one individual are indicated as well as total loci obtained across all individuals. Total number of variable SNPs (Var), parsimony informative SNPS (PI), and unlinked bi-allelic SNPs (BI) are also included

\begin{tabular}{|c|c|c|c|c|c|c|c|c|c|c|c|c|c|}
\hline \multirow[t]{2}{*}{ (m) } & \multirow[t]{2}{*}{ (c) } & \multicolumn{6}{|c|}{ Number of Loci } & \multicolumn{6}{|c|}{ Number of SNPs } \\
\hline & & Min & Max & Total & Min & Max & Total & Var. & $\mathrm{Pl}$ & $\mathrm{Bl}$ & Var. & $\mathrm{Pl}$ & $\mathrm{BI}$ \\
\hline Clade 4 & & & p10 & & & p25 & & & p10 & & & $p 25$ & \\
\hline \multirow[t]{2}{*}{1.0} & 0.90 & 73 & 73 & 73 & 154 & 154 & 154 & 382 & 78 & 63 & 1,358 & 617 & 144 \\
\hline & 0.85 & 93 & 93 & 93 & 185 & 185 & 185 & 563 & 255 & 83 & 1,675 & 778 & 175 \\
\hline \multirow[t]{2}{*}{0.75} & 0.90 & 1,570 & 2,391 & 2,552 & 3,139 & 5,331 & 5,515 & 17,690 & 10,505 & 2,448 & 48,301 & 28,211 & 5,411 \\
\hline & 0.85 & 1,869 & 2,869 & 2,958 & 3,791 & 6,113 & 6,343 & 22,936 & 14,026 & 2,851 & 60,232 & 36,467 & 6,236 \\
\hline \multirow[t]{2}{*}{0.50} & 0.90 & 3,391 & 9,742 & 10,813 & 6,923 & 24,178 & 26,861 & 70,048 & 38,239 & 10,490 & 214,870 & 121,983 & 26,532 \\
\hline & 0.85 & 4,347 & 10,341 & 11,540 & 8,693 & 25,221 & 28,179 & 86,607 & 49,558 & 11,225 & 251,615 & 147,779 & 27,860 \\
\hline \multicolumn{14}{|c|}{ Clade $5 C$} \\
\hline \multirow[t]{2}{*}{1.0} & 0.90 & 115 & 115 & 115 & 143 & 143 & 143 & 885 & 212 & 104 & 1,222 & 356 & 132 \\
\hline & 0.85 & 123 & 123 & 123 & 154 & 154 & 154 & 908 & 229 & 109 & 1,325 & 385 & 140 \\
\hline \multirow[t]{2}{*}{0.75} & 0.90 & 2,500 & 4,174 & 4,306 & 4,060 & 6,675 & 7,083 & 52,325 & 31,844 & 4,276 & 91,810 & 57,263 & 7,051 \\
\hline & 0.85 & 2,781 & 4,793 & 4,968 & 4,491 & 7,771 & 8,060 & 62,057 & 38,113 & 4,930 & 106,548 & 66,726 & 8,022 \\
\hline \multirow[t]{2}{*}{0.50} & 0.90 & 6,210 & 11,587 & 13,189 & 9,439 & 20,926 & 23,946 & 171,091 & 104614 & 13141 & 329,837 & 205,990 & 23,898 \\
\hline & 0.85 & 5,381 & 11,697 & 13,281 & 9,329 & 20,582 & 23,484 & 175,558 & 108,258 & 13,225 & 327,211 & 205,557 & 23,428 \\
\hline
\end{tabular}




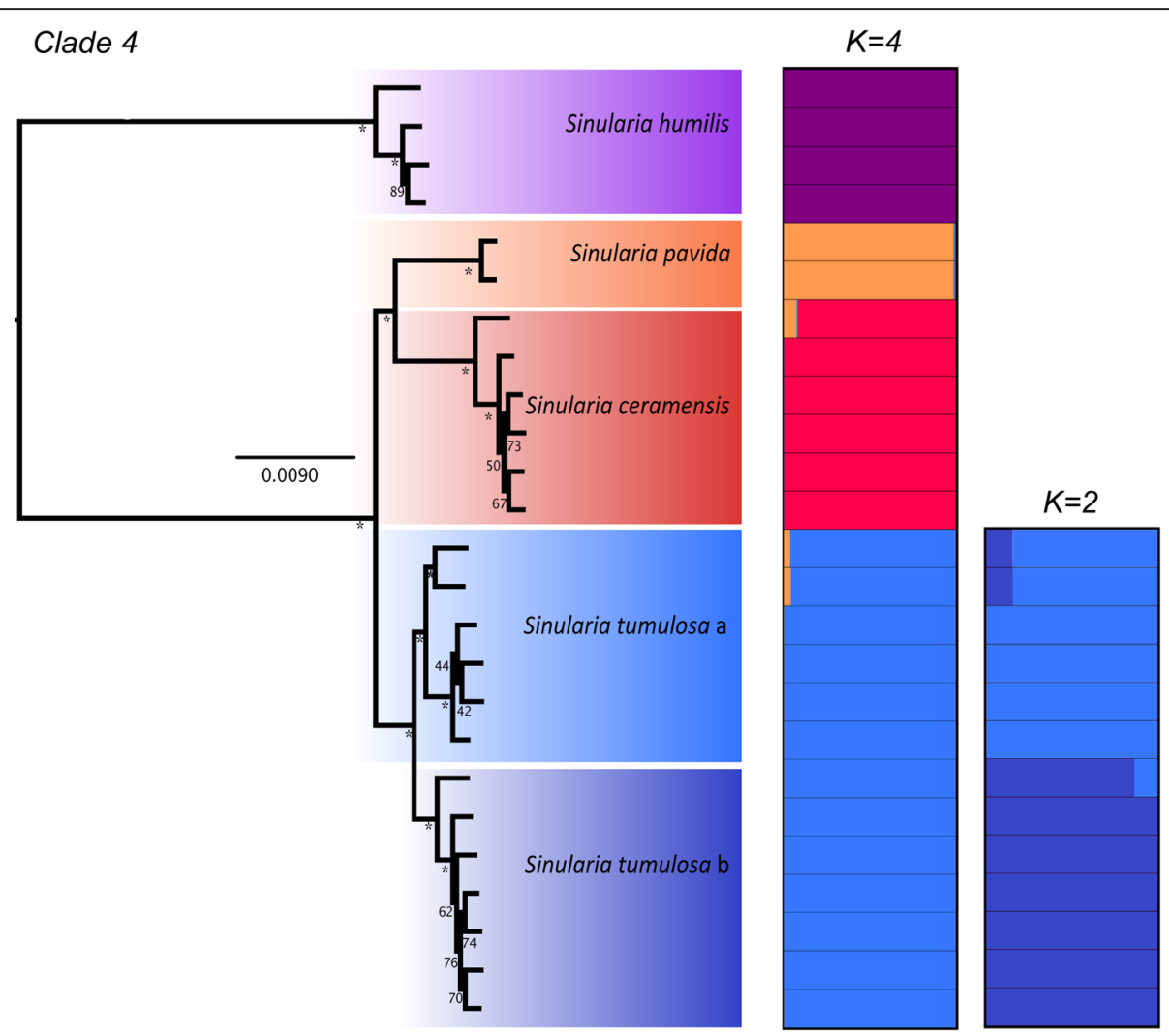

Fig. 2 Maximum likelihood phylogeny of Dongsha Atoll Sinularia clade 4 constructed using RAxML rapid bootstrapping (200 b.s. replicates) on the concatenated c 0.85, m 0.75, p 0.25 locus dataset. * denotes 100\% b.s. support. Distruct plots are included and show the probability of individual membership into different $K$ clusters ( $K=4$ for clade 4 and $K=2$ for the $S$. tumulosa group). Colors denote different species. $S$. tumulosa is designated as ' $a$ ' and ' $b$ ' as results strongly suggest this clade consists of two species

support this result. First, following a one-species model $(\mathrm{MLE}=-1119), \mathrm{DAPC}+1$ was the second most likely $(\mathrm{MLE}=-1291)$ species model according to BFD* analyses (Table 3). The DAPC+ 1 model included species denoted by DAPC, plus two sub-clades of $S$. tumulosa. Second, most of the individuals of these two sub-clades formed two separate groupings in the DAPC plot, although there was some overlap among individuals (Fig. 5a). Finally, S. tumulosa was divided into two well-supported, reciprocally monophyletic clades (sp. a and b) in both concatenated and species tree phylogenies, which match the $m t M u t S$ and combined mtMutS $+28 S$ results (Figs. 1a, 2 and 4a; Additional files 1: Figures S1 and S2). It is possible that these represent two cryptic species.

Twenty-four separate ABBA-BABA tests were performed on clade 4 (Fig. 6, Additional file 2). The average number of loci shared across taxa in each test was $3313 \pm$ 556 (Additional file 3). The ABBA-BABA tests indicated admixture between $S$. tumulosa and $S$. pavida lineages $(\alpha=3.0, Z=3.44-4.10, D=0.11$; tests 10,18$)$. Eleven of thirteen individuals of $S$. tumulosa (both clades a and b) appeared to be strongly admixed with S. pavida $(\alpha=3.0$, $\mathrm{Z}=3.11-4.55, \quad \mathrm{D}=0.11-0.18$; tests $11,14-17,19-24$,
Additional file 3). Upon further examination with partitioned D-statistics, introgression appeared to have occurred from S. pavida into both S. tumulosa clades $(\alpha=$ $3.0, \mathrm{Z}=2.8-3.0, \mathrm{D}=-0.14-0.15$, Additional file 3).

\section{Clade 5C}

DAPC and Structure analyses supported eight species in Sinularia clade 5C (S. acuta/abrupta, S. densa, S. exilis, S. lochmodes, S. maxima, S. penghuensis/daii, S. slieringsi, and S. wanannensis); S. abrupta was not distinguished from $S$. acuta, and the holotype of $S$. daii was placed with S. penghuensis. In addition, five individuals identified as $S$. penghuensis, including the holotype and paratypes, grouped with S. slieringsi. The optimal number of $K$ clusters suggested by the DAPC analyses was eight $(\mathrm{BIC}=377$, Additional file 1: Figure S3), and the DAPC plot revealed no overlap among these distinct clusters (Fig. 5b). Further support for group assignment can be seen in the assignment plots, as all individuals were successfully re-assigned into their respective clusters (Additional file 1: Figure S5). In addition, the Distruct plot clearly illustrated little to no admixture between these eight species (Fig. 3), except that both individuals of $S$. exilis appeared to be admixed with at 


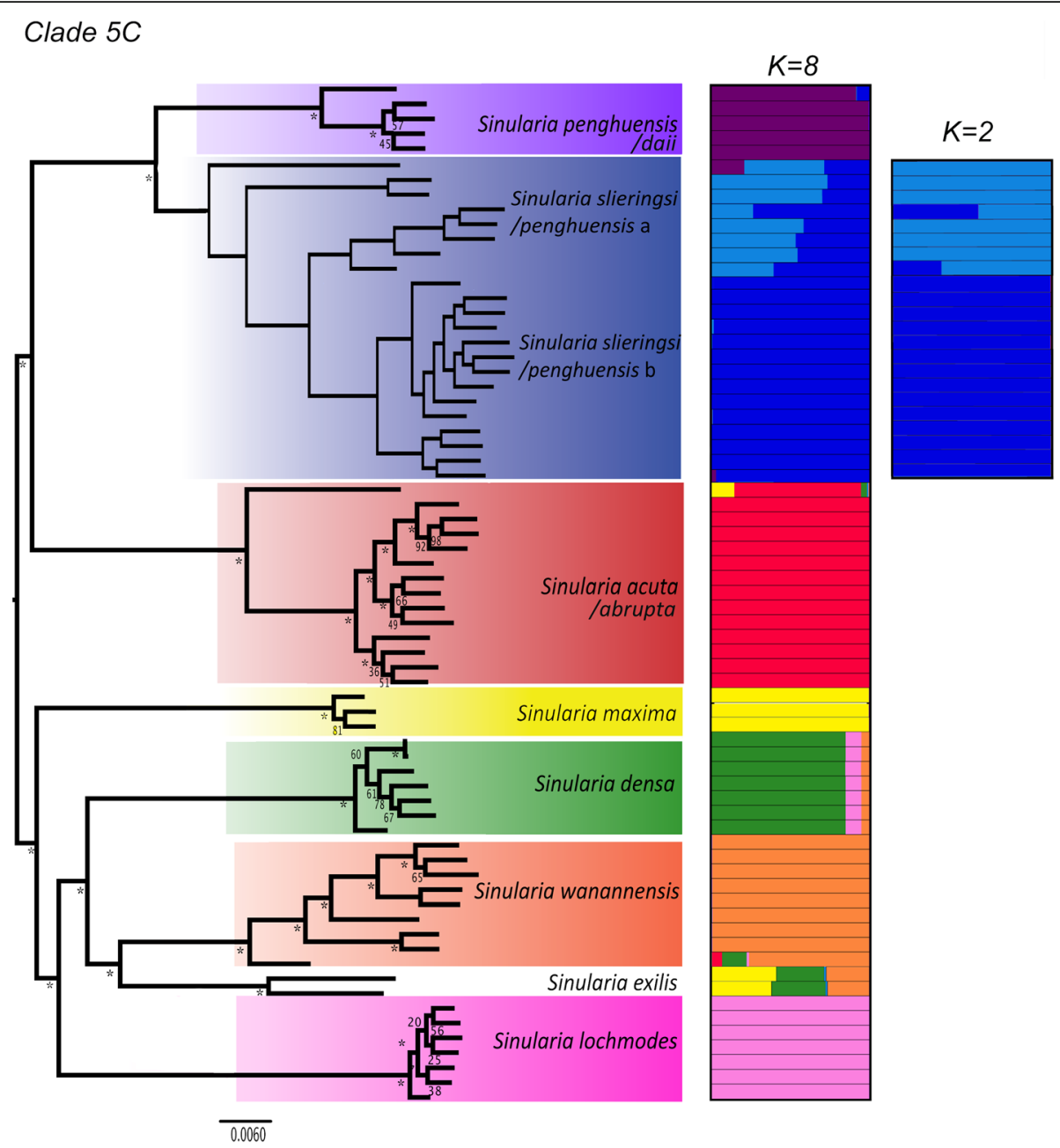

Fig. 3 Maximum likelihood phylogeny of Dongsha Atoll Sinularia clade 5C constructed using RAxML rapid bootstrapping (200 b.s. replicates) on the concatenated c 0.85, m 0.75, p 0.25 locus dataset. * denotes 100\% b.s. support. Distruct plots are included and show the probability of membership into different $K$ clusters ( $K=8$ for clade $5 C$, and $K=2$ for the S. slieringsi/penghuensis group). Colors denote different species. S. slieringsi/penghuensis is designated as ' $a$ ' and ' $b$ ' as results suggest this clade may consist of two species

least three different species, including S. densa, S. maxima, and S. wanannensis. Individuals of S. abrupta (D329) and the holotype of S. wanannensis (Z34695) whose $28 S$ barcode sequences were incongruent with their $m t M u t S$ haplotypes (Additional file 1: Figure S1) also showed some evidence of admixture with $S$. maxima and $S$. densa, respectively.

It is possible that $S$. slieringsi represents two cryptic species, although results are not conclusive. Upon further Structure analysis, little to no admixture was revealed between two sub-clades of S. slieringsi; however, two individuals (one of them a paratype of S. penghuensis, Z34681) appeared admixed (Fig. 3). Following a one-species model $(\mathrm{MLE}=-1298)$, DAPC+ 1 was the second most likely (MLE $=-1543)$ species model according to BFD* analyses (Table 3 ). The DAPC+ 1 model included species designated by DAPC, plus the two groups of S. slieringsi. Second, individuals of S. slieringsi formed two separate groupings in the DAPC plot, although there was some overlap among individuals (Fig. 5b). S. slieringsi also split into two reciprocally monophyletic clades in the species tree phylogeny (Fig. 4b), but not in the concatenated RAxML or $m t M u t S+28 S$ phylogenies (Fig. 3; Additional file 1: Figure S2).

Fifty separate ABBA-BABA tests were run on clade $5 \mathrm{C}$ (Fig. 7, Additional file 2). The average number of loci shared across taxa in each test was $1745 \pm 216$ (Additional file 3). The ABBA-BABA tests indicated admixture between the $S$. penghuensis/S. daii clade and one clade, clade $\mathrm{b}$, of $S$. slieringsi $(\alpha=3.0, \mathrm{Z}$ scores $=4.51, \mathrm{D}=-0.15$; test 14). Most individuals in the latter clade (which included the holotype of $S$. penghuensis) appeared to be strongly admixed as evidenced by ABBA-BABA tests $(\alpha=3.0, \mathrm{Z}=$ $3.1-5.5, \mathrm{D}=-0.13--0.20$; tests $15,19-26,28)$. As suggested 


\section{a Clade 4}

S. humilis

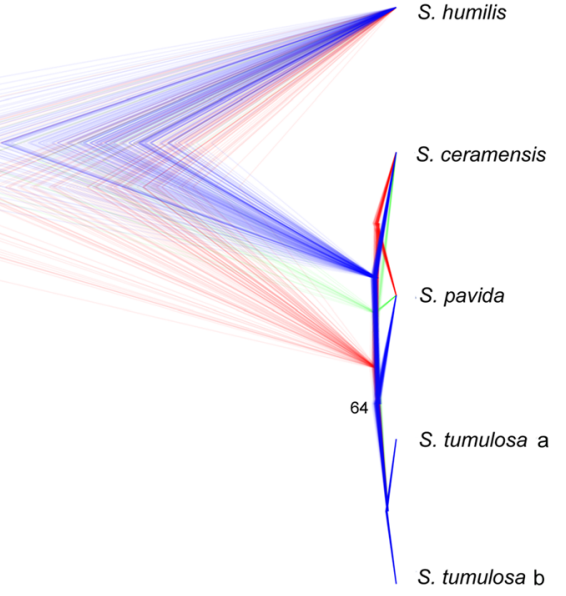

\section{b Clade $5 \mathrm{C}$}

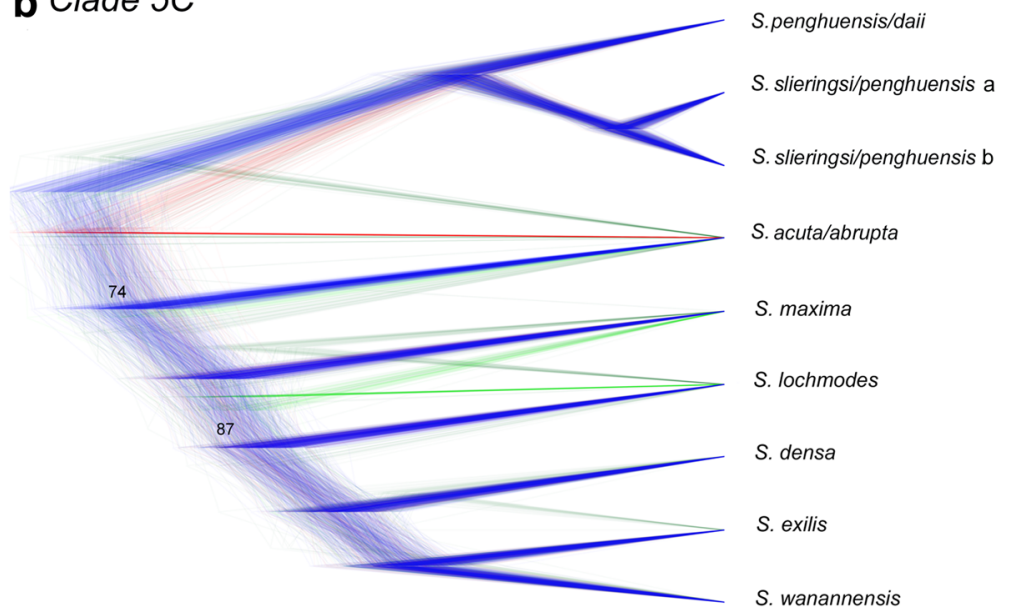

Fig. 4 Species trees of Dongsha Atoll Sinularia clades 4 and 5C. Cloudograms illustrate the best species delimitation models (DAPC +1$)$ for both clades inferred from bi-allelic SNP data [a clade 4: 6,236 SNPs and $\mathbf{b}$ clade 5C: 8,022 SNPs); m0.75 datasets] using SNAPP species tree analyses. The maximum clade credibility tree and congruent trees are in blue. Trees with different topologies are in red and green. Posterior probabilities at internal nodes $>95 \%$ unless indicated
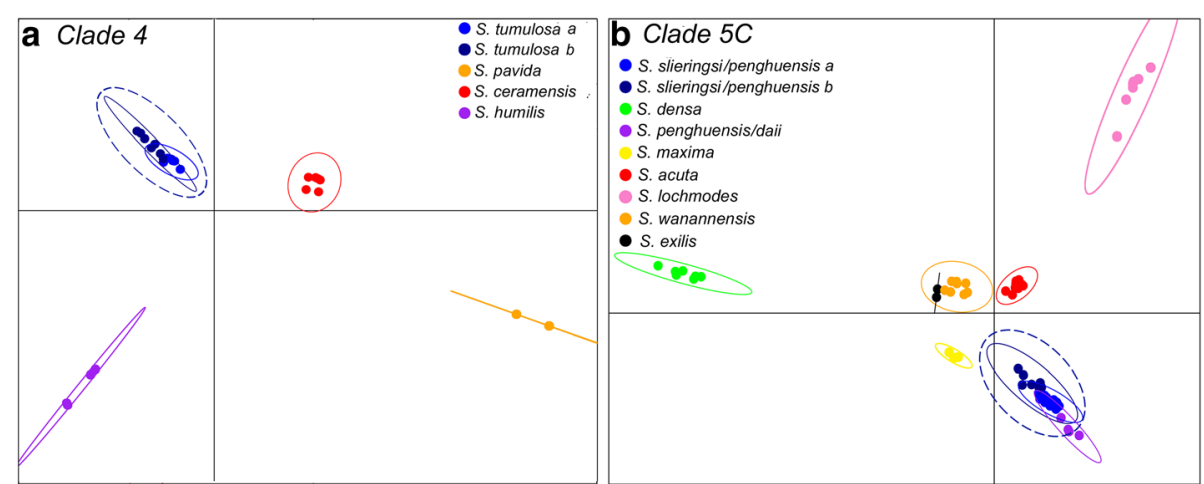

Fig. 5 Discriminant analysis of principal components (DAPC) plots for Dongsha Atoll Sinularia a clade 4 and $\mathbf{b}$ clade 5C. Genetic clusters representing different morphospecies are color coded to match the phylogenetic trees in Figs. 2, 3 and 4. Species (S. tumulosa in (a) and S. slieringsi/penghuensis in (b) encircled in dotted lines) that were suggested to be further divided into two species by Bayes Factor Determination are also denoted 
Table 3 SNAPP results for different species delimitation models for Dongsha Atoll Sinularia clades 4 and $5 C$

\begin{tabular}{llll}
\hline Model & MLE & BF & Rank \\
\hline Clade 4 & & - & 1 \\
ONESP & -1119 & -662 & 6 \\
TWOSPP & -1450 & -416 & 5 \\
THREESPP & -1327 & -362 & 3 \\
mtMutS & -1300 & -382 & 4 \\
DAPC/28 s & -1310 & -344 & 2 \\
DAPC+ 1 & -1291 & & \\
Clade 5C & & -- & 1 \\
ONESP & -1298 & -724 & 7 \\
TWOSPP & -1660 & -592 & 4 \\
FOURSPP & -1594 & -654 & 6 \\
mtMutS & -1625 & -618 & 5 \\
28 S & -1607 & -492 & 3 \\
DAPC & -1544 & -490 & 2 \\
DAPC+ 1 & -1543 &
\end{tabular}

Rank of most likely species model based on Bayes Factor delimitation is indicated

MLE Marginal Likelihood Estimate, BF Bayes Factor

by the Structure analysis, the holotype of $S$. wanannensis (Z34695) showed strong admixture with $S$. densa $(\alpha=$ 3.0, $\mathrm{Z}=3.13, \mathrm{D}=0.14$; test 9$)$, but not with $S$. maxima $(\alpha=$ $3.0, Z=0.25, D=-0.01$; test 2 ). It was also not admixed with either S. penghuensis or S. slieringsi $(\alpha=3.0, \mathrm{Z}=0.24-0.61$,
$\mathrm{D}=-0.03-0.01$; tests $46-47)$, even though it shared the same $m t M u t S$ haplotype as both of those species. Although S. abrupta D329 showed some evidence of admixture with $S$. maxima and $S$. densa in the Structure analysis, the ABBA-BABA tests indicated that it was not a hybrid $(\alpha=$ $3.0, \mathrm{Z}=1.59-2.78, \mathrm{D}=0.10-0.18$; tests 32,45$)$. The $S$. abrupta specimen, Z33623, that shared a $28 S$ sequence with $S$. densa was also not significantly admixed with that species $(\alpha=3.0, Z=2.11, D=0.13$, test 44$)$, whereas $S$. abrupta $\mathrm{D} 19$ was $(\alpha=3.0, \mathrm{Z}=312, \mathrm{D}=0.15$; test 40$)$. One individual of $S$. acuta, D450, was also admixed with $S$. densa $(\alpha=3.0, Z=3.21, D=0.16$; test 37$)$. Notably, the Distruct plot showed strong admixture of the two $S$. exilis specimens with $S$. densa, S. maxima, and $S$. wanannensis (Fig. 3). However, ABBA-BABA tests suggested that these individuals were not significantly introgressed with those or any other species $(\alpha=3.0, Z=0.25-2.96, D=-0.15-0.04$; tests $0-2,5-6,8,48-49)$. Overall, the ABBA-BABA tests indicated that at least 15 individuals were significantly introgressed with other species at an $\alpha=3.0$; however, we note that $\mathrm{D}$ statistics for several other tests also deviated considerably from 0 , although they were not significant at $\alpha=3.0$.

\section{Discussion}

\section{Species delimitations}

A few different species delimitation methods based on RADseq data resulted in incongruence in the number of species suggested to be present among those sampled at

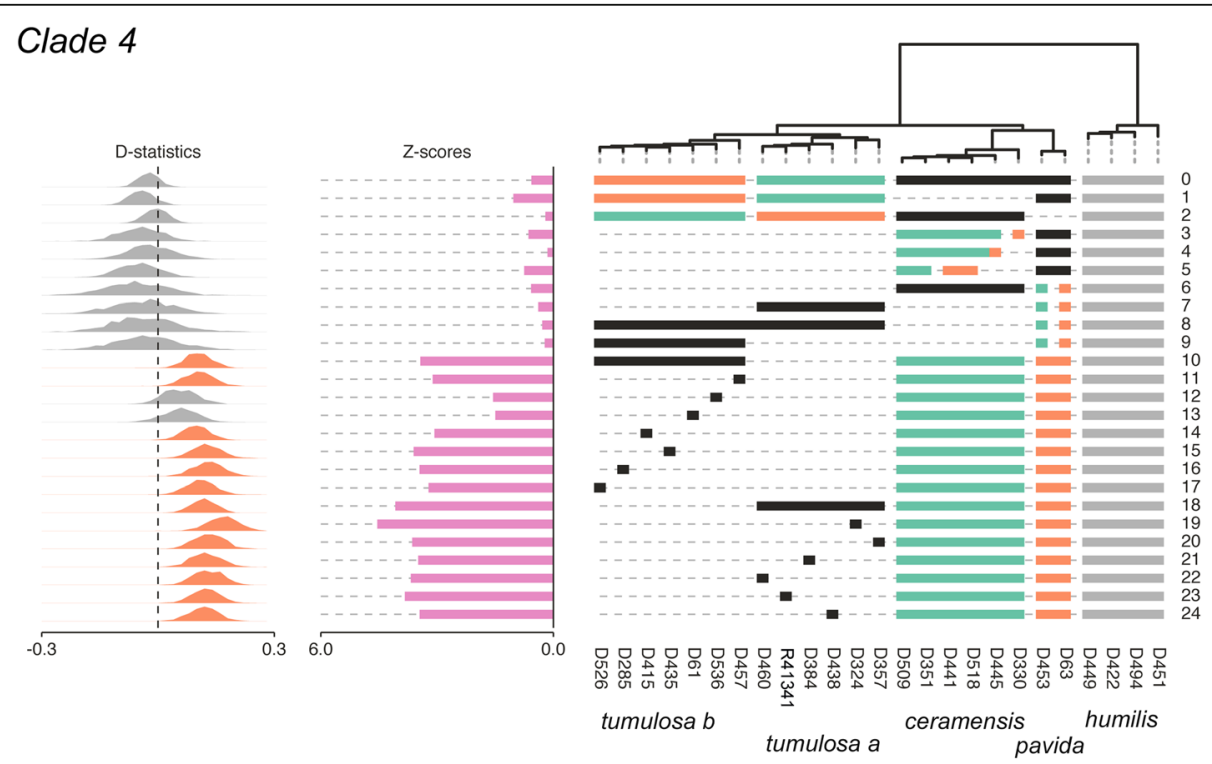

Fig. 6 D-statistic tests for admixture in Dongsha Atoll Sinularia clade 4. Test numbers are listed on the right for each 4-taxon test (((p1, p2), p3), p4). Horizontal bars below the tips of the tree indicate which taxa were included in each test. S. humilis was set as the outgroup for all tests (indicated by gray bars). Tests are configured to ask whether P3 (black bars) shares more derived SNPs with lineage P1 (green bars) relative to P2 (orange bars). As illustrated to the left, Z scores are bar plots and D-statistics are histograms. Histograms are green for significant gene flow between P1 and P3 (BABA) and orange for significant gene flow between P2 and P3 (ABBA). D-statistics that were not significant are gray. Significance was assessed at an alpha level of 3.0 (i.e., when $D$ deviates $>3.0$ standard deviations from zero) 


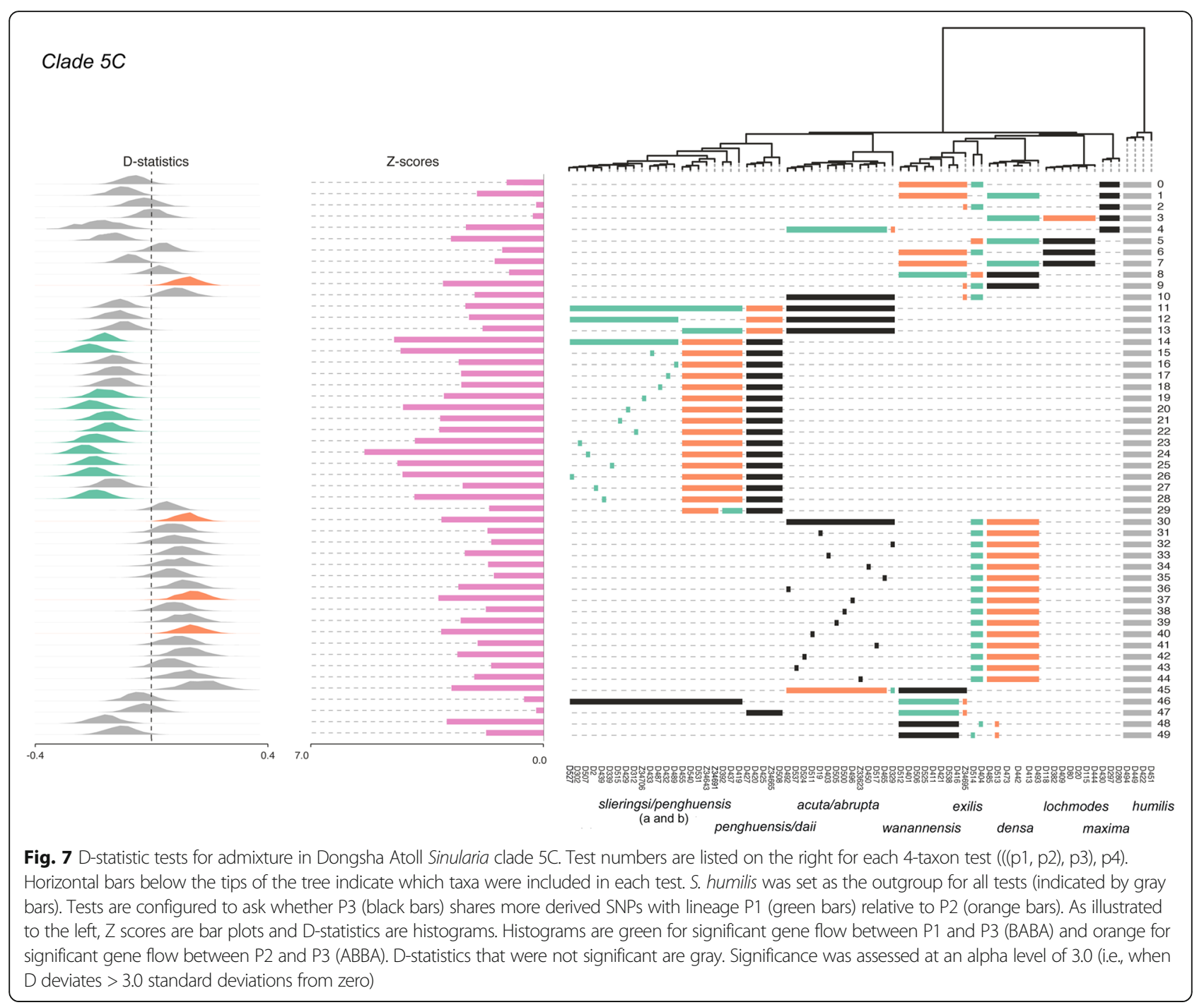

Dongsha Atoll in each of Sinularia clades 4 and 5C. BFD* analysis indicated that for each clade the most likely model was a one-species model, whereas DAPC indicated that four species were present in clade 4 and eight were present in clade $5 \mathrm{C}$. It seems unlikely that only a single species exists in each of clades 4 and clade $5 \mathrm{C}$ because there is little overlap among groups in the DAPC plots and there is strong genetic structure shown in the Structure analyses. Furthermore, there are well-supported clades in both ML and species trees, many of them congruent with distinct morphologies [8]. The suggestion by BFD* analysis of only one species in each of the two clades is likely spurious due to the relatively few loci included in those analyses. Because of the computational time it took to run each SNAPP species-delimitation model, only complete datasets were used, i.e., those that included no missing data, and these contained relatively few SNPs $(<200)$.

All other species delimitation analyses supported four or five species of Sinularia in clade 4 and eight or nine in clade $5 \mathrm{C}$ among the samples that were sequenced. Within clade 4, RADseq and both barcoding markers discriminated S. humilis, S. ceramensis and S. pavida from one another and from all specimens identified as S. tumulosa. Both the $m t M u t S+28 S$ barcode and RADseq results further delineated two distinct clades within $S$. tumulosa, with hybridization tests suggesting that both are admixed with S. pavida. Within clade 5C, species delimitation analyses clearly distinguished S. lochmodes, S. densa, S. wanannensis and $S$. maxima from all other species, and each of those morphospecies also had unique haplotypes at both barcoding loci. S. acuta was similarly delineated from all other morphospecies with the notable exception of $S$. abrupta. Two of three individuals of S. abrupta shared a mtMutS haplotype with $S$. acuta, and could not be distinguished from that species in the DAPC and Structure analyses. A third colony identified as S. abrupta (D329) showed signs of admixture in both Structure and ABBA-BABA analyses, suggesting a possible hybrid origin. As $S$. acuta and $S$. 
abrupta differ markedly in both colony growth form and sclerite morphology [8], the apparent lack of genetic distinction between these two morphospecies warrants further study.

The remaining morphospecies in clade $5 \mathrm{C}$ could not be separated clearly using either barcode marker, and the multilocus analyses suggested that admixture might contribute to the difficulty distinguishing them. Although both individuals of $S$. exilis had unique $m t M u t S$ haplotypes, they shared $28 S$ genotypes with $S$. densa and S. wanannensis. Phylogenetic analyses placed $S$. exilis as the sister to $S$. wanannensis, but Structure suggested considerable admixture with both $S$. maxima and $S$. densa. As ABBA-BABA tests did not strongly support a hybrid origin of $S$. exilis, incomplete lineage sorting may better explain why this species shares genotypes with other species in the clade. Hybridization was, however, supported as a possible explanation for the confusing relationship between S. penghuensis and S. slieringsi, two morphospecies that shared several different mtMutS haplotypes, one of which was also shared by $S$. daii. Five colonies of $S$. penghuensis, including the holotype (ZMTAU Co 34706) and two paratypes (ZMTAU Co 34643 , Co 34681), shared a $28 S$ genotype with S. slieringsi, while the other four shared a very different $28 \mathrm{~S}$ genotype with the holotype of $S$. daii (ZMTAU Co 34665). Multilocus species delimitation analyses separated the latter four S. penghuensis plus $S$. daii from a large clade that included all S. slieringsi plus the S. penghuensis type specimens. Structure and DAPC further separated that large clade into two sub-clades, suggestive of possible cryptic species. ABBA-BABA tests indicated that a majority of the individuals of $S$. slieringsi and $S$. penghuensis in one of those two sub-clades are admixed with the S. penghuensis-S. daii clade.

\section{Evidence for hybridization in Sinularia}

It is important to use a phylogenetic framework in assessments of introgressive hybridization. A species that appears admixed could have a close relative harboring a stronger signal of admixture [26], and if that species is not included in analyses, then the admixture will be incorrectly attributed to a closely-related taxon [23, 25, 90]. In addition, it can be challenging to distinguish introgression between two species from "secondary genomic admixture", which occurs when one species shares recent ancestry with a true hybridizing lineage, thus causing that species to also appear as if it were admixed $[25,26]$. Although the current study sequenced most of the clade 4 and 5C morphospecies that occur at Dongsha Atoll, there were at least two morphospecies in each clade that were not included in the analyses. In addition, there are other Sinularia species in phylogenetically distinct clades that also inhabit this atoll, and those too were not included in the analyses. While our results provide evidence for hybridization, we acknowledge the possibility of incorrectly attributing admixture to a close relative of the true hybridizing lineage, as not all possible Sinularia morphospecies were included in these analyses.

Because of the difficulties interpreting ABBA-BABA tests even when using a phylogenetic framework [26], it is best to focus on the strongest signals of admixture [28]. The signal of introgression was strong in clade 4, with both clades of $S$. tumulosa showing admixture with $S$. pavida; no other hybridization tests in this clade were significant, with D-statistics centered around zero and $\mathrm{Z}$ scores fairly low. In contrast, it was more difficult to confidently determine which Sinularia species are hybridizing with others in clade $5 \mathrm{C}$, which could be due to the use of an incomplete phylogeny. There were some cases where the signal of admixture was strong (e.g., S. acuta with $S$. densa, S. slieringsi with $S$. penghuensis) and supported by other tests in addition to ABBA-BABA. However, there were also cases where species appeared admixed, but the ABBA-BABA results were not significant at an alpha of 3.0. For example, Structure analyses suggested there was considerable admixture between $S$. wanannensis and $S$. exilis, and at least one $S$. exilis individual had the same $28 S$ barcode as $S$. wanannensis, but the ABBA-BABA tests were not significant. Perhaps with more S. exilis individuals in the analyses, or with the addition of the missing morphospecies of Sinularia, a more complete picture would emerge as to whether these species share genes as a result of incomplete lineage sorting or hybridization. Because the current phylogenetic analysis did not include all species, it is possible that the species identified as the source of introgressed alleles may simply be close relatives of the actual parental species. Nevertheless, the phylogenetic framework was a useful approach in determining that hybridization appears to be an important process in the diversification of this speciose group of soft corals.

Incongruence between the RAxML and SNAPP species trees (both built using 25\% missing data) may provide further support for hybridization among Sinularia species. In the clade 4 SNAPP species tree, S. ceramensis was sister to a clade of $S$. pavida plus $S$. tumulosa, whereas in the ML tree built using concatenated RAD loci, S. ceramensis was sister to S. pavida. In clade 5C, S. acuta was sister to $S$. penghuensis and S. slieringsi in the ML tree, but sister to all other species in the SNAPP species tree. Notably, relationships that differed between analyses showed evidence of admixture in the ABBA-BABA results. Although incomplete lineage sorting can lead to discordance between phylogenies built using concatenated data vs. species tree methods $[29,61,66]$, hybridization has also been shown to produce incongruence among gene trees [29, 62]. Introgressive hybridization may also explain the alternative topologies recovered in the SNAPP species tree. Johnston 
et al. [53] suggested that alternative trees emerging from SNAPP analyses of corals in the genus Porites could be due to introgressive hybridization, incomplete lineage sorting, or contamination of loci by symbionts (e.g., Symbiodiniaceae). The species that displayed different relationships in the SNAPP trees were shown to be potentially hybridizing by our ABBA-BABA tests, suggesting that alternative SNAPP topologies may be driven by the presence of introgressive hybridization. Results further lend support to the idea that diversification of species-rich lineages may not be a solely bifurcating process. As such, phylogenetic tree reconstructions that include taxa that do not follow the usual assumption of a bifurcating process of evolution can lead to incongruence among gene trees and contribute to difficulties in resolving phylogenies.

\section{Morphospecies versus genetic data}

Incongruence between morphological and molecular evidence for species boundaries is common in corals (e.g., $[36,37,58])$. Contributing factors include environmental plasticity (e.g., [80]) and frequent homoplasy of morphological characters (e.g., [36]), as well as the slow rate of mitochondrial gene evolution that has made "universal" molecular barcodes such as COI relatively invariant among congeneric species $[50,71,96]$. When barcodes fail to discriminate distinct morphospecies it may be because the markers lack appropriate variation, or, alternatively, because morphological variation within a species has been incorrectly interpreted as evidence of a species boundary [73]. Attempts to integrate the two different sources of evidence have met with some success, as demonstrated by Benayahu et al. [8]. By combining assessment of morphology with a character-based barcoding approach, that study identified at least 27 species of Sinularia from Dongsha Atoll, including those used in the current study. In several cases in which distinct morphotypes shared identical $m t M u t S$ haplotypes, however, the lack of congruence was attributed to invariance of the barcode marker (i.e., incomplete lineage sorting), and thus species were delimited using morphological characters. In two such cases, species delimitation methods using RADseq data also failed to support the genetic distinction between discrete morphotypes, namely S. acuta and S. abrupta, and S. slieringsi and S. penghuensis. Moreover, our inclusion of type specimens of $S$. penghuensis and $S$. daii as taxonomic references revealed no genetic distinction between the material identified here as S. slieringsi and the S. penghuensis types, or between the holotype of $S$. daii and additional colonies identified as $S$. penghuensis. In addition, a colony from Palau identified as S. verruca (R41341) could not be distinguished genetically from one of the two clades of S. tumulosa. Clearly, additional taxonomic work integrating both morphological and molecular approaches will be necessary to clarify the relationships among these taxa.
Morphological discrimination of species is complicated in Sinularia and many other soft corals due to the continuous nature of many of the characters used to diagnose species. Colony growth forms and the intricate shapes of sclerites are difficult to quantify and may present a continuum of variation, as do morphometric characters such as the sizes of sclerites commonly reported in the literature (e.g. [111]). Many of the species examined here, including S. tumulosa, $S$. verruca, $S$. acuta and $S$. daii, were described from single exemplars $[6,67,76]$, and therefore no data exist on the possible range or limits of morphological variation they exhibit, which potentially confounded efforts to discriminate them from other similar species. Hybridization also offers a possible explanation for the lack of congruence between morphological and molecular determinations of species identity. As has been suggested for some coral genera, hybridization can lead not only to morphologically distinct or intermediate phenotypes (Porites: [38]; Acropora: [107, $109,112])$, but also to F1 hybrids that exhibit characters of both parental species [101, 112]. In a naturally occurring hybrid zone in Guam, for example, F1 hybrids of $S$. maxima and S. polydactyla were found to contain a mix of sclerites resembling those of both parental species [101]. Perhaps mechanisms such as these add to the confusion in morphospecies identification, which is often pervasive in Sinularia and other octocorals. Although it is currently unknown whether or not hybridization generates new species or asexual lines in the genus Sinularia, admixture between Sinularia species occurs, and perhaps contributes to the range of morphotypes observed in the genus.

\section{Utility of DNA barcoding in corals}

As numerous other studies have now cautioned, none of the single-gene molecular barcodes currently used to help guide species identifications in octocorals successfully delimit all species [3, 48, 71, 72, 79], particularly when genetic distance thresholds are used to decide species boundaries. For example, in Sinularia clade 5C only four MOTUs were identified among eight morphospecies using the $28 \mathrm{~S}$ marker, whereas mtMutS resolved eight MOTUs, but not all of them were congruent with morphospecies and RADSeq delimitations. Such lack of concordance among different molecular markers is not uncommon in corals and in recently diverged clades more generally [72, 81, 85, 108]. For octocorals, a consensus has emerged that mitochondrial and $r D N A$ barcodes may be useful in species assessments for some taxa (e.g., $[72,79])$, but not all. Multiple markers as well as other lines of evidence need to be considered when delimiting species [73]. Genomic approaches such as RADseq are effective [48, 79], but still prohibitively expensive and impractical to use for the routine species identification work required of biodiversity surveys. Alternatively, once species boundaries have been validated 
using such approaches, it may be possible to identify morphological or simple molecular characters that are species-diagnostic. As discussed above, however, the continuum of variation in morphological traits of corals complicates the search for diagnostic characters, and in some recently discriminated octocoral taxa none have yet been identified [73].

Single-gene barcode markers such as mtMutS and $28 \mathrm{~S}$ offer diagnostic nucleotide characters that can be used to identify cryptic taxa [71]. When Benayahu et al. [8] applied a character-based $m t M u t S$ barcode to the Sinularia species found at Dongsha, the only morphospecies that could not be discriminated were the same ones for which the current study also found incongruence between RAD clades and morphospecies designations: S. acuta, S. abrupta, S. penghuensis, S. daii and S. slieringsi. A compound, characterbased barcode that combines mtMutS with 28S, however, yields diagnostic characters that discriminate each of the Sinularia clades identified by RADseq, including both sub-clades of S. tumulosa and S. slieringsi (Additional file 1: Figure S6). Once species boundaries have been validated using integrated, genomic approaches, such as those applied here, use of simple character-based barcodes to identify morphologically cryptic species may be more timeand cost-effective than genomic approaches. Assignment of character-based barcodes, however, requires a priori recognition of species boundaries, as well as screening of a sufficient number of individuals to identify polymorphic characters.

\section{Future research directions}

Further investigation is needed to determine the evolutionary processes responsible for generating the high species diversity in the genus Sinularia, but a necessary first step is to understand how many species there are and where they are distributed. With accurate species identifications utilizing both classical taxonomy and advanced genomic techniques, it will be possible to address questions pertaining to how and when Sinularia diversified into coral reef environments and why species in this genus appear to be so successful at co-existing. One intriguing question is whether the high diversity of Sinularia was generated in sympatry through mechanisms such as hybrid speciation or whether species have diverged in allopatry and then colonized the same reefs. With the advent of new genomic techniques such as RADSeq and target-capture genomics (e.g., [83]), we can begin to examine how pervasive hybridization is on coral reefs, particularly because F1 hybrids and their progeny may be more fit than the parent populations, and hybrid vigor may help in the maintenance and resilience of coral reef diversity [101]. Using a genomic approach can help to guide species delimitation while simultaneously shedding light on the processes generating diversity in this genus- just one of many hyperdiverse coral lineages (e.g., the scleractinians Acropora, Porites, Pocillopora and the octocorals Dendronephthya, Lobophytum and Sarcophyton) in which ecologically similar congeners co-occur in high numbers on coral reefs throughout the Indo-Pacific.

\section{Conclusions}

The results presented here suggest that the answer to both of the questions that originally motivated this study is 'yes'. In most-but not all-cases, multilocus species delimitation analyses validated the species boundaries between morphologically distinct taxa that nonetheless shared identical mtMutS or $28 S$ barcode sequences. In some cases, barcodes appeared to be shared among morphospecies as a result of incomplete lineage sorting at mitochondrial or rDNA loci. In other cases, including ones in which multilocus analyses did not support the genetic distinction of individuals that differed morphologically, there was evidence for admixture and introgressive hybridization among species. Finally, in several cases in which morphospecies could not be distinguished genetically but there was no evidence for admixture or hybridization, it will be necessary to validate whether or not the observed morphological differences are indicative of species boundaries or intraspecific variation.

Delimiting species is a critical first step in documenting the biodiversity of ecologically important reef inhabitants, such as species in the genus Sinularia. This study demonstrates the utility of using genomic approaches to delimit species within a hyperdiverse lineage of soft corals and to examine simultaneously whether hybridization may be contributing to its diversification. Although there was some incongruence among datasets and species delimitation methods, we can confidently conclude that the sequenced individuals represent at least four species of Sinularia in clade 4 and eight species in clade $5 \mathrm{C}$ [70], with the potential for one additional cryptic species in each clade. The results point to hybridization as an evolutionary pathway to diversification in Sinularia, and suggest that this mechanism may produce hybrids with morphologies intermediate to those of their parental species, contributing to the difficulty of assigning species based on morphology in this and other coral genera [38, 40, 74, 101]. Furthermore, our results raise the possibility that hybrid speciation (i.e., reticulate evolution via introgressive hybridization) is one mechanism that has contributed to the diversity of octocorals.

\section{Methods}

Specimen collection and preparation

Colonies of Sinularia were collected using SCUBA during biodiversity surveys conducted at Dongsha Atoll Marine 
National Park (Taiwan) in 2011, 2013 and 2015 [8]. Collections were made at 13 sites in the shallow fore-reef zone (3-21 m) surrounding the $25-\mathrm{km}$ diameter atoll (see Fig. 1 in [8]). During the 2015 survey we specifically targeted common morphospecies belonging to clades 4 and $5 \mathrm{C}$ [70]. Wedges of tissue (usually $<50 \mathrm{~cm}^{2}$ in area) were cut from the colony perimeter and pried off the substrate; colonies typically heal and regenerate this lost tissue within a few months (pers. obs.). Following collection, small subsamples of tissue $(\sim 100 \mathrm{mg})$ were preserved in $95 \% \mathrm{EtOH}$, and the remainder of the specimen was preserved in $70 \%$ EtOH. All vouchers have been deposited in the Steinhardt Museum of Natural History, Tel Aviv University, Israel (ZMTAU, Additional file 4). To identify morphospecies, sclerites were isolated from colonies by dissolving tissue in $10 \%$ sodium hypochlorite and examined using either light microscopy or, when necessary, scanning electron microscopy (SEM) [8]. The morphological IDs were made by direct comparison to type material when available. Specimens belonging to 13 Sinularia morphospecies, four from clade 4 and nine from clade $5 \mathrm{C}$, were selected for further species delimitation analyses. Seven specimens collected in previous biodiversity surveys of the Penghu Archipelago, Taiwan [7] and Palau [72], including original type material of $S$. penghuensis (ZMTAU Co 34643 (=Z34643), Co 34706 (= Z34706), Co 34681 (=Z34681)), S. wanannensis (ZMTAU Co 34695 (=Z34695)) and $S$. daii (ZMTAU Co 34665 (= Z34665)), were also included as taxonomic references (Table 1; Additional file 4).

DNA was extracted from 95 Sinularia specimens, and quantified using a Qubit v 2.0 fluorometer (Broad Range Assay Kit). Quality was assessed by running $100 \mathrm{ng}$ of DNA for each sample on a $1 \%$ agarose gel, and checked with a NanoDrop spectrophotometer. Concentration of high-quality (230/260 and 260/280 ratios > 1.8) DNA was normalized to $20 \mathrm{ng}$ per $\mathrm{ul}$ and sent to Floragenex Inc. (Eugene, OR) for RADSeq library preparation. DNA libraries were constructed for each of the 95 samples using the 6-cutter PstI enzyme, and then split into two for sequencing $100 \mathrm{bp}$ SE reads on two full lanes of an Illumina HiSeq2500 (University of Oregon's Genomics and Cell Characterization Core Facility lab). In addition to RADseq, two gene regions ( $m t M u t S, 28 S \mathrm{rDNA}$ ) used widely for barcoding in octocorals were PCR-amplified and Sanger-sequenced using published primers and protocols $[71,72]$.

\section{Phylogenetic inference and species delimitation using DNA barcodes}

$m t M u t S$ and $28 \mathrm{~S}$ sequences of the Sinularia species were each aligned using the L-INS-i method in MAFFT [56], and pairwise genetic distances (Kimura 2-parameter) among sequences were calculated using the DNADist program in PHYLIP v. 3.69 [35]. MOTHUR v 1.29 [94] was used to delimit molecular operational taxonomic units (MOTUs) based on a genetic distance threshold of $0.3 \%$ applied to $m t M u t S$ and $28 \mathrm{~S}$ separately as well as combined in a concatenated dataset (e.g., [72]). Phylogenetic trees were constructed separately for $m t M u t S$ and $28 S \mathrm{rDNA}$ and for the concatenated dataset using maximum likelihood methods (Garli; [117]) (Additional file 1: Figures S1 and S2). jModelTest [19] was used to identify the best models of evolution (AIC criterion) to use in these analyses (mtMutS: $\mathrm{HKY}+\mathrm{G} ; 28 S$ rDNA: HKY + I).

\section{Phylogenetic inference and species delimitation using RADSeq data}

We produced several different RADSeq locus datasets using pyRAD v 3.0 [24] with different parameter combinations, following recommendations in the pyRAD and ipyRAD tutorials [24, 27] and previous studies [48, 79]. Datasets chosen for species delimitation and phylogenetic analyses included those loci from pyRAD parameter settings that maximized the number of phylogenetically informative sites, while minimizing missing data and eliminating the potential for paralogous loci (Table 2, see Additional file 5 for more details). Thus, for most analyses, we used data produced from the following parameter settings: $c 0.85, p 0.25$, and $m 0.75$ for each of clades 4 and $5 \mathrm{C}$.

RAxML v8 [102] was used to create maximum likelihood (ML) phylogenies for clades 4 and 5C. A GTR + G+I model as suggested by the Akaike Information Criterion [(AIC = 371918), JModelTest v2, [19]] was used. A total of $20 \mathrm{ML}$ searches and 200 bootstrap replicates were performed using rapid bootstrapping on concatenated loci. RAxML analyses were performed (12 analyses per clade) for each of the different datasets produced by pyRAD with different parameter combinations and clustering thresholds (Table 2, Additional files 5 and 6).

Discriminant Analysis of Principal Components (DAPC) was performed using the package 'adegenet' v2.0 in $\mathrm{R}[54,84]$ to explore genetic structure in both clades 4 and $5 \mathrm{C}$. The DAPC method, used previously in species delimitation analyses [79], forms clusters based on genetic similarity of each multilocus genotype, without considering a model of evolution. We first used the function find.clusters to find the best number of $K$ genetic clusters in unlinked SNP datasets for each clade. Find.clusters was performed on $K=20$ for each clade. The lowest value of the Bayesian Information Criterion (BIC) statistic was used to detect the optimal number of $K$ clusters. These clusters were then analyzed using DAPC, which first transforms the data using principal components analysis and then performs a Discriminant Analysis on the retained principal components. The optim.a.score function was used to determine how many $\mathrm{PC}$ axes needed to be retained (Additional file 1: Figure 
S7). Six PCAs and four discriminant functions were retained for clade 4; and six PCAs and six discriminant functions were retained for clade $5 \mathrm{C}$ (Additional file 1: Figure S8). Scatterplots of discriminant functions were then created. We also used the function assignplot to visualize individual membership in each $K$ cluster, which can help show the accuracy of the cluster assignments and identify any individuals that have high probabilities of membership in $>1$ cluster.

The Bayesian model-based clustering approach, Structure v2.3 [82], was also used to infer the number of Sinularia species. The program clusters individuals based on genetic variation alone, without any other prior information such as geographic origin or population assignment. Structure was performed on unlinked SNP datasets for both Sinularia clades 4 and 5C, and run in parallel using StrAutoParallel v 1.0 [16] using an admixture model with correlated allele frequencies. Burnin was set to 250,000 followed by 1,000,000 MCMC generations. The inferred number of populations $(K)$ was set from 1 to 8 for clade 4 and 1 to 10 for clade 5C; 20 runs of each $K$ were conducted. Multiple runs of each $K$ were aligned with CLUMPP v 1.2 [51], and the resulting indivq file was input into Distruct v. 1.1 [91] for graphical display of individual population assignments. The commonly used $\Delta K$ method [30] was not used in our study to identify an optimal $K$ value because this method is known to be most successful at finding only the uppermost levels of genetic structure in a hierarchical system [30]. Initial tests of the $\Delta K$ method revealed $K=$ 2 as the best model for each clade; however, analysis at $K>2$ indicated strong genetic structure in both clades 4 and $5 \mathrm{C}$. Therefore, we plotted the population structure assignments of $K=4$ for clade 4 and $K=8$ for clade 5C, because these were the number of genetic clusters suggested by DAPC analyses, and Structure analyses indicated little to no admixture between putative species at these $K$ clusters. Then, following Gowen et al. [42], we analyzed successively smaller groups of potential species in separate analyses at $K=2$. We plotted the results of $K=2$ runs for the putative species, $S$. tumulosa and $S$. slieringsi, because Bayes Factor Delimitation with genome data (BFD*) analyses (see below), suggested the presence of additional species within each of S. tumulosa and S. slieringsi.

Coalescent-based SNAPP v 1.3 [13] analyses were used to test alternative species models for both clades 4 and $5 \mathrm{C}$. Samples were assigned to the following alternative species models (Additional file 1: Figures S9 and S10): 1) one species (ONESP), 2) two species (TWOSPP), 3) DAPC clusters (DAPC), 4) DAPC clusters plus division of another clade (DAPC+1), 5) MOTU assignments based on $m t M u t S$ (MUTS), and 6) MOTU assignments based on $28 S$ rDNA (28S). In addition, three (THREESPP) and four
(FOURSPP) species models were also tested for clades 4 and $5 \mathrm{C}$, respectively. SNAPP analyses were performed in BEAST v 2.4.5 [11] with a path sampling of 48 steps (MCMC length $=100,000$, pre-burnin $=1000)$ following Leaché et al. [64] and Herrera \& Shank [48]. The $c 0.85$, $m 1.0, p 0.25$ bi-allelic SNP datasets (175 SNPs for clade 4, 140 SNPs for clade $5 \mathrm{C}$, no missing data) were used because of the long computational time it took for each SNAPP run. Marginal likelihood estimates were obtained for each different model run in SNAPP analyses. The different species delimitation models were then ranked using BFD* methods. Bayes Factors were calculated between each of two alternative models by subtracting the marginal likelihood estimates between two models, and then multiplying the difference by two (following $[57,64]$ ).

SNAPP was also used to infer the species tree for each Sinularia clade. Three independent runs were performed on SNP data $($ MCMC length $=1,000,000$, pre-burnin $=$ 1000 , samplefreq $=1000)$ using BEAST with default parameters for mutation rate, coalescent rate, and ancestral population sizes (following [48]). The c0.85, $m 0.75$, $p 0.25$ bi-allelic SNP datasets were used for species tree analyses. Acceptance probabilities were checked to ensure that tuning parameters were appropriate and the chain mixed well [22]. Log files were combined using Log Combiner v 1.1 and input into Tracer v1.6 [86]. Convergence and ESS $>200$ were assessed using Tracer after a $10 \%$ burnin. Maximum clade credibility trees were generated with Tree Annotator v 2.3 [11]. Both the consensus tree and all tree topologies were drawn in DensiTree v2.2 [10].

\section{Hybridization tests}

We calculated Patterson's D statistics in ipyRAD v. 0.7.28 [27] to test for hybridization between species. Briefly, these tests calculate the proportion of $\mathrm{ABBA}$ and $\mathrm{BABA}$ site patterns, and excess of either is indicative of admixture rather than incomplete lineage sorting $[23,43]$. Multiple 4-taxon tests were generated for both clades 4 and 5C (Additional file 2). For both clades, $S$. humilis was set as the outgroup ('p4'). For tests that included multiple individuals per lineage, SNP frequencies were pooled. For tests performed on clade 4, each species was set as 'p3' and all possible 4-species combinations were tested. For clade $5 \mathrm{C}$, all possible 4 -species combinations were tested in each of two sub-clades (i.e., the S. slieringsi-S. penghuensis-S. acuta clade and the S. wanannensis- S. exilis-S. lochmodes-S. densa-S. maxima clade). Additional tests were conducted either when Structure results indicated potential admixture or there was incongruence between the different molecular markers (i.e., 28S, mtMutS, RAD). When test results were significant at the species level, further tests were performed to determine if particular individuals within the lineage were admixed. Significance of 
each test was determined by performing 1000 bootstrap replicates in which loci were resampled with replacement. Both D statistics and Z statistics, which represent the number of bootstrap standard deviations (alpha $=3.0)$ that D statistics deviate from zero [34], are reported. Following D-statistic tests, partitioned D-statistic tests were performed for a few cases to examine the direction of introgression. Tests were conducted and figures were plotted following the ipyRAD ABBA-BABA cookbook in Jupyter Notebook [60].

\section{Additional files}

Additional file 1: Figure S1. ML trees of barcode data. ML phylogenies generated using mtMutS and $28 \mathrm{~S}$ rDNA barcode data. Figure S2. ML tree of combined barcode data. ML phylogeny generated using a mtMutS + 28S rDNA dataset. Figure S3. BIC plots. Bayesian Information Criteria indicating optimal number of K clusters. Figure S4. Probability of membership plot for Clade 4. Individual reassignments based on discriminant functions. Figure S5. Probability of membership plot for Clade 5C. Individual reassignments based on discriminant functions. Figure S6. Character based barcodes. Species-diagnostic nucleotide characters. Figure S7. Optimal a-scores. A-scores indicate the optimal number of principal components to retain. Figure S8. Discriminant analysis eigenvalues. Total number of discriminant eigenvalues retained. Figure S9. Clade 4 species models and RAxML phylogeny. Species models used in BFD* analysis with phylogeny constructed using RAXML on RADSeq data. Figure S10. Clade 5C species models and RAXML phylogeny. Species models used in BFD* analysis with phylogeny constructed using RAxML on RADSeq data. (ZIP $22000 \mathrm{~kb}$ )

Additional file 2: ABBA-BABA tests. List of ABBA-BABA tests performed in ipyRAD. (TXT $13 \mathrm{~kb}$ )

Additional file 3: ABBA-BABA test results. Results of ABBA-BABA tests for both clades 4 and 5C. (XLSX $65 \mathrm{~kb})$

Additional file 4: Sinularia specimen data. Table of specimen data with morphospecies identifications and assigned species delimitations based on MOTUs and RADSeq data. (XLSX $19 \mathrm{~kb}$ )

Additional file 5: Supplemental Methods. Description of additional methods $[15,63,65,95,104,110]$. (PDF $79 \mathrm{~kb})$

Additional file 6: Concatenated nexus files of all phylogenies constructed using RAxML on different pyRAD RADSeq datasets. (TXT $141 \mathrm{~kb}$ )

\section{Abbreviations}

AFLP: Amplified fragment length polymorphism; BEAST: Bayesian evolutionary analysis by sampling trees; BFD*: Bayes factor delimitation (* with genomic data); DAPC: Discriminant analysis of principal components; DNA: Deoxyribonucleic acid; FOURSPP: Four species; MCMC: Markov chain Monte Carlo; MLE: Marginal likelihood estimate; MOTU: Molecular operational taxonomic unit; mtMutS: Mitochondrial mutS-like protein; ONESP: One species; PC: Principal components; PCA: Principal components analysis; RADSeq: Restriction siteassociated sequencing; RAxML: Randomized axelerated maximum likelihood; rDNA: Ribosomal deoxyribonucleic acid; SNAPP: SNP and AFLP package for phylogenetic analysis; SNP: Single nucleotide polymorphism; THREESPP: Three species; TWOSPP: Two species; ZMTAU Co: Zoological Museum Tel Aviv University, Coelenterates

\section{Acknowledgements}

We thank the staff members of Dongsha Atoll National Park, Dongsha Atoll Research Station (DARS), and Biodiversity Research Center, Academia Sinica (BRCAS) for assistance during the field work; A. Gonzalez, J.M. Adams, A. TorresNavarro, and N. Liu for help with DNA extraction and barcoding; Ben Titus for helpful discussions; and Alex Shlagman (Steinhardt Museum of Natural History) for curatorial skills.

\section{Funding}

This study was made possible by a grant to YB from the Taiwanese Ministry of Science (MOST) to conduct octocoral surveys at Dongsha Atoll. Additional funding was provided by Dongsha Atoll Research Station, Howard Hughes Medical Institute Undergraduate Science Education Program award \#52006301 to Harvey Mudd College, and by NSF-DEB \#1457817 to C.S. McFadden. These funding agencies played no role in the design of the study; collection, analysis, and interpretation of data or in writing the manuscript.

\section{Availability of data and materials}

Raw RADSeq data: SRA accession: PRJNA529740

Mafft alignments, RAxML trees, and Jupyter notebook hosted on Open

Science Framework, DOI: https://doi.org/10.17605/OSF.IO/SW4YZ

28 s sequences: Genbank \#s MK333539-MK333628, KF915525

mtMutS sequences GenBank \#s: See Additional file 4

\section{Authors' contributions}

CSM, TW, AMQ and YB conceived and designed the study. KS and M-SJ coordinated logistics for field work, including obtaining funding and collection permits. CSM and YB collected specimens. AMQ and CSM conducted all phylogenetic and species delimitation analyses, and drafted the manuscript TW prepared samples, sequenced barcode genes and conducted preliminary RADSeq analyses. YB made morphological identifications. CSM, TW, AMQ, YB, KS and M-SJ all revised and approved the final version of this manuscript.

\section{Ethics approval and consent to participate}

All collections were made in accordance with permits obtained from Marine National Parks, Taiwan.

\section{Consent for publication}

Not Applicable

\section{Competing interests}

The authors declare that they have no competing interests.

\section{Publisher's Note}

Springer Nature remains neutral with regard to jurisdictional claims in published maps and institutional affiliations.

\section{Author details}

${ }^{1}$ Biology Department, Harvey Mudd College, 1250 N. Dartmouth Ave, Claremont, CA 91711, USA. ${ }^{2}$ Institute of Marine Biology, National Sun Yat-sen University, Kaohsiung, Taiwan. ${ }^{3}$ Biodiversity Research Center, Academia Sinica, Taipei, Taiwan. ${ }^{4}$ School of Zoology, George S. Wise Faculty of Life Sciences, Tel Aviv University, 69978 Ramat Aviv, Israel.

Received: 16 January 2019 Accepted: 23 April 2019

Published online: 06 June 2019

\section{References}

1. Aratake S, Tomura T, Saitoh S, Yokokura R, Kawanishi Y, Shinjo R, Reimer JD, Tanaka J, Maekawa H. Soft coral Sarcophyton (Cnidaria: Anthozoa: Octocorallia) species diversity and chemotypes. PLoS One. 2012;7:e30410.

2. Babcock RC, Bull GD, Harrison PD, Heyward AJ, Oliver JK, Wallace CC, Willis BL. Synchronous spawnings of 105 scleractinian coral species on the great barrier reef. Mar Biol. 1986;90:379-94.

3. Baco AR, Cairns SD. Comparing molecular variation to morphological species designations in the deep-sea coral Narella reveals new insights into seamount coral ranges. PLoS One. 2012;7:e45555.

4. Benayahu Y, Loya Y. Space partitioning by stony corals, soft corals and benthic algae on the coral reefs of the northern Gulf of Eilat (Red Sea). Helgoländer Meeresun. 1977;30:362-82.

5. Benayahu Y. Species composition of soft corals (Octocorallia, Alcyonacea) on the coral reefs of Sesoko Island, Ryukyu archipelago, Japan. Galaxea. 1995;12:103-24.

6. Benayahu Y, van Ofwegen LP. New species of octocorals (Coelenterata: Anthozoa) from the Penghu archipelago, Taiwan. Zool Stud. 2011;50:350-62

7. Benayahu Y, van Ofwegen LP, Soong K, Dai CF, Jeng MS, Shlagman A, Hsieh HJ, McFadden CS. Diversity and distribution of Octocorals (Coelenterata: Anthozoa) on the coral reefs of Penghu, Taiwan. Zool Stud. 2012;51:1529-48. 
8. Benayahu Y, van Ofwegen LP, Dai CF, Jeng MS, Soong K, Shlagman A, et al. The octocorals of Dongsha Atoll (South China Sea): an iterative approach to species identification using classical taxonomy and molecular barcodes. Zool Stud. 2018:57. https:/doi.org/10.6620/Zs.2018.57-50.

9. Blunt JW, Carroll AR, Copp BR, Davis RA, Keyzers RA, Prinseps MR. Marine natural products. Nat Prod Rep. 2018;35:8-53.

10. Bouckaert RR. DensiTree: making sense of sets of phylogenetic trees. Bioinformatics. 2010;26:1372-3.

11. Bouckaert R, Heled J, Kühnert D, Vaughan T, Wu CH, Xie D, Drummond AJ. BEAST 2: a software platform for Bayesian evolutionary analysis. PLoS Comp Biol. 2014;10(4): e1003537.

12. Bruno JF, Siddon CE, Witman JD, Colin PL, Toscano MA. El Niño related coral bleaching in Palau Western Caroline Islands. Coral Reefs. 2001;20:127-36.

13. Bryant D, Bouckaert R, Felsenstein J, Rosenberg NA, RoyChoudhury A. Inferring species trees directly from biallelic genetic markers: bypassing gene trees in a full coalescent analysis. Mol Biol Evol. 2012;29:1917-32.

14. Carlo JM, Barbeitos MS, Lasker HR. Quantifying complex shapes: elliptical Fourier analysis of octocoral sclerites. Biol Bull. 2011;220:224-37.

15. Catchen J, Hohenlohe PA, Bassham S, Amores A, Cresko WA. Stacks: an analysis tool set for population genomics. Mol Ecol. 2013;22:3124-40.

16. Chhatre VE, Emerson KJ. StrAuto: automation and parallelization of STRUCTURE analysis. BMC Bioinf. 2017;18:192.

17. Combosch DJ, Vollmer SV. Trans-Pacific RAD-Seq population genomics confirms introgressive hybridization in eastern Pacific Pocillopora corals. Mol Phylogenet Evol. 2015;88:154-62

18. Currat M, Ruedi M, Petit R, Excoffier $L$. The hidden side of invasions: massive introgression by local genes. Evolution. 2008;62:1908-20.

19. Darriba D, Taboada GL, Doallo R, Posada D. jModelTest 2: more models new heuristics and parallel computing. Nat Methods. 2012;9:772.

20. Diekmann O, Bak R, Stam W, Olsen J. Molecular genetic evidence for probable reticulate speciation in the coral genus Madracis from a Caribbean fringing reef slope. Mar Biol. 2001;139:221-33.

21. Dinesen ZD. Patterns in the distribution of soft corals across the great barrier reef. Coral Reefs. 1983;1:229-36.

22. Drummond AJ, Bouckaert RR. Bayesian evolutionary analysis with BEAST. UK: Cambridge University Press; 2015.

23. Durand EY, Patterson N, Reich D, Slatkin M. Testing for ancient admixture between closely related populations. Mol Biol Evol. 2011;28:2239-52.

24. Eaton DA. PyRAD: assembly of de novo RADseq loci for phylogenetic analyses. Bioinformatics. 2014;30:1844-9.

25. Eaton DA, Ree RH. Inferring phylogeny and introgression using RADseq data: an example from flowering plants (Pedicularis: Orobanchaceae). Syst Biol. 2013;62:689-706.

26. Eaton DA, Hipp AL, González-Rodríguez A, Cavender-Bares J. Historical introgression among the American live oaks and the comparative nature of tests for introgression. Evolution. 2015;69:2587-601.

27. Eaton DAR, Overcast I. ipyRAD: interactive assembly and analysis of RADseq data sets; 2016.

28. Eaton DA. Ipyrad Cookbook. 2018. https:/nbviewer.jupyter.org/github/dereneaton/ ipyrad/blob/master/tests/cookbook-abba-baba.ipynb. Accessed 20 Dec 2018

29. Edwards SV, Liu L, Pearl DK. High-resolution species trees without concatenation. PNAS. 2007;104:5936-41

30. Evanno G, Regnaut S, Goudet J. Detecting the number of clusters of individuals using the software STRUCTURE: a simulation study. Mol Ecol. 2005;14:2611-20.

31. Fabricius KE. Reef invasion by soft corals: which taxa and which habitats? In: Greenwood JG, Hall NJ, editors. Proceedings of the Australian Coral Reef Society 75th Anniversary Conference. Heron Island. October 1997: University of Queensland, Brisbane; 1998. p. 77-90.

32. Fabricius K. Tissue loss and mortality of soft corals following mass-bleaching. Coral Reefs. 1999;18:54.

33. Fabricius K, Alderslade P. Soft corals and sea fans: a comprehensive guide to the tropical shallow-water genera of the central West-Pacific the Indian Ocean and the Red Sea. Australian Institute of Marine Science: Townsville; 2001.

34. Federman S, Donoghue MJ, Daly DC, Eaton DA. Reconciling species diversity in a tropical plant clade Canarium Burseraceae. PLoS One. 2018; 13(6):e0198882.

35. Felsenstein J. PHYLIP phylogeny inference package Distributed by the author. Department of Genome Sciences University of Washington Seattle Version 3; 2005.
36. Forsman ZH, Barshis DJ, Hunter CL, Toonen RJ. Shape-shifting corals: molecular markers show morphology is evolutionarily plastic in Porites. BMC Evol Biol. 2009;9:45.

37. Forsman ZH, Concepcion GT, Haverkort RD, Shaw RW, Maragos JE, Toonen RJ. Ecomorph or endangered coral? DNA and microstructure reveal Hawaiian species complexes: Montipora dilatata/flabellata/turgescens M. patula/verrilli. PLoS One. 2010;5:e15021.

38. Forsman ZH, Knapp ISS, Tisthammer K, Eaton DAR, Belcaid M, Toonen RJ. Coral hybridization or phenotypic variation? Genomic data reveal gene flow between Porites lobata and P. compressa. Mol Phyl. Evol. 2017;111:132-48.

39. Frade PR, Reyes-Nivia MC, Faria J, Kaandorp JA, Luttikhuizen PC, Bak RPM. Semipermeable species boundaries in the coral genus Madracis: introgression in a brooding coral system. Mol. Phyl. Evol. 2010;57:1072-90.

40. Fukami H, Budd AF, Levitan DR, Jara J, Kersanach R, Knowlton N. Geographic differences in species boundaries among members of the Montastraea annularis complex based on molecular and morphological markers. Evolution. 2004;58:324-37.

41. Goulet TL, LaJeunesse TC, Fabricius KE. Symbiont specificity and beaching susceptibility among soft corals in the 1998 great barrier reef mass coral bleaching event. Mar Biol. 2006;154:795-804.

42. Gowen FC, Maley JM, Cicero C, Peterson AT, Faircloth BC, Warr TC, McCormack JE. Speciation in Western scrub-jays Haldane's rule and genetic clines in secondary contact. BMC Evol Biol. 2014;14:135.

43. Green RE, Krause J, Briggs AW, Maricic T, Stenzel U, Kircher M, Hansen NF. A draft sequence of the Neandertal genome. Science. 2010;328:710-22.

44. Harrison PL, Babcock RC, Bull GD, Oliver JK, Wallace CC, Willis BL. Mass spawning in tropical reef corals. Science. 1984;223:1186-9.

45. Hatta M, Fukami H, Wang W, Omori M, Shimoike K, Hayashibara T, Sugiyama T. Reproductive and genetic evidence for a reticulate evolutionary history of mass-spawning corals. Mol Biol Evol. 1999;16:1607-13.

46. Hebert PD, Penton EH, Burns JM, Janzen DH, Hallwachs W. Ten species in one: DNA barcoding reveals cryptic species in the neotropical skipper butterfly Astraptes fulgerator. PNAS. 2004a;101:14812-7.

47. Hebert PD, Stoeckle MY, Zemlak TS, Francis CM. Identification of birds through DNA barcodes. PLoS Biol. 2004b;2:e312.

48. Herrera S, Shank TM. RAD sequencing enables unprecedented phylogenetic resolution and objective species delimitation in recalcitrant divergent taxa. Mol Phyl Evol. 2016;100:70-9.

49. Hickerson MJ, Meyer CP, Moritz C. DNA barcoding will often fail to discover new animal species over broad parameter space. Syst Biol. 2006;55:729-39.

50. Huang D, Meier R, Todd PA, Chou LM. Slow mitochondrial COI sequence evolution at the base of the metazoan tree and its implications for DNA barcoding. J Mol Evol. 2008:66:167-74.

51. Jakobsson M, Rosenberg NA. CLUMPP: a cluster matching and permutation program for dealing with label switching and multimodality in analysis of population structure. Bioinformatics. 2007; 23:1801-6.

52. Jeng M-S, Huang H-D, Dai C-F, Hsiao Y-C, Benayahu Y. Sclerite calcification and reef-building in the fleshy octocoral genus Sinularia (Octocorallia: Alcyonacea). Coral Reefs. 2011;30:925-33.

53. Johnston EC, Forsman ZH, Flot JF, Schmidt-Roach S, Pinzón JH, Knapp IS, Toonen RJ. A genomic glance through the fog of plasticity and diversification in Pocillopora. Sci Rep. 2017;7:5991.

54. Jombart $\mathrm{T}$, Collins C. A tutorial for discriminant analysis of principal components DAPC using adegenet. Imperial College of London MRC Center for Outbreak Analysis and Modelling; 2015.

55. Kahng SE, Benayahu Y, Lasker HR. Sexual reproduction in octocorals. Mar Ecol Prog Ser. 2011;443:265-83.

56. Katoh $\mathrm{K}$, Toh $\mathrm{H}$. Recent developments in the MAFFT multiple sequence alignment program. Brief Bioinform. 2008;9:286-98.

57. Kass RE, Raftery AE. Bayes factors. J Am Stat Assoc. 1995;90:773-95.

58. Keshavmurthy S, Yang S-Y, Alamaru A, Chuang Y-Y, Pichon M, Obura D, Chen AC. DNA barcoding reveals the coral "laboratory rat" Stylophora pistillata encompasses multiple identities. Sci Rep. 2013;3:1520.

59. Kim E, Lasker HR, Coffroth MA, Kim K. Morphological and genetic variation across reef habitats in a broadcast-spawning octocoral. Hydrobiologia. 2004;530:423-32.

60. Kluyver T, Ragan-Kelley B, Pérez F, Granger BE, Bussonnier M, Frederic J, Ivanov $P$, et al. Jupyter notebooks-a publishing format for reproducible computational workflows: ELPUB; 2016;87-90. 
61. Kubatko LS, Degnan JH. Inconsistency of phylogenetic estimates from concatenated data under coalescence. Syst Biol. 2007;56:17-24.

62. Kubatko LS. Identifying hybridization events in the presence of coalescence via model selection. Syst Biol. 2009;58:478-88.

63. LaJeunesse TC, Parkinson JE, Gabrielson PW, Jeong HJ, Reimer JD, Voolstra CR, Santos SR. Systematic revision of Symbiodiniaceae highlights the antiquity and diversity of coral endosymbionts. Curr Biol. 2018;28:2570-80

64. Leaché AD, Fujita MK, Minin VN, Bouckaert RR. Species delimitation using genome-wide SNP data. Syst Biol. 2014;63:534-42.

65. Lin S, Cheng S, Song B, Zhong X, Lin X, Li W, Cai M. The Symbiodinium kawagutii genome illuminates dinoflagellate gene expression and coral symbiosis. Science. 2015;350:691-4.

66. Maddison WP. Gene trees in species trees. Syst Biol. 1997:46:523-36

67. Manuputty AEW, van Ofwegen LP. The genus Sinularia (Octocorallia: Alcyonacea) from Ambon and Seram Moluccas Indonesia. Zoologische Mededelingen Leiden. 2007:81:187-216.

68. Marshall PA, Baird AH. Bleaching of corals on the great barrier reef: differential susceptibilities among taxa. Coral Reefs. 2000;19:155-63.

69. McFadden CS, Hutchinson MB. Molecular evidence for the hybrid origin of species in the soft coral genus Alcyonium (Cnidaria: Anthozoa: Octocorallia). Mol Ecol. 2004;13:1495-505.

70. McFadden CS, van Ofwegen LP, Beckman E, Benayahu Y, Alderslade P. Molecular systematics of the speciose indo-Pacific soft coral genus Sinularia (Anthozoa: Octocorallia). Invertebr Biol. 2009;128:303-23.

71. McFadden CS, Benayahu Y, Pante E, Thoma JN, Nevarez PA, France SC. Limitations of mitochondrial gene barcoding in Octocorallia. Mol Ecol Resour. 2011;11:19-31.

72. McFadden CS, Brown AS, Brayton C, Hunt CB, van Ofwegen LP. Application of DNA barcoding to biodiversity studies of shallow-water octocorals: molecular proxies agree with morphological estimates of species richness in Palau. Coral Reefs. 2014;33:275-86.

73. McFadden CS, Haverkort-Yeh R, Reynolds AM, Halàsz A, Quattrini AM, Forsman ZH, Toonen RJ. Species boundaries in the absence of morphological ecological or geographical differentiation in the Red Sea octocoral genus Ovabunda (Alcyonacea: Xeniidae). Mol Phylogenet Evol. 2017:112:174-84.

74. Miller DJ, Van Oppen MJ. A 'fair go' for coral hybridization. Mol Ecol. 2003; 12:805-7.

75. van Ofwegen LP. Status of knowledge of the Indo-Pacific soft coral genus Sinularia May 1898 (Anthozoa: Octocorallia). Proc 9th Intl Coral Reef Symp. 2002;1:167-71.

76. van Ofwegen LP. The genus Sinularia (Octocorallia: Alcyonacea) at Palau Micronesia. Zoologische Mededelingen Leiden. 2008;82:631-735.

77. van Ofwegen LP, Benayahu Y, McFadden CS. Sinularia leptoclados Ehrenberg 1834 Cnidaria: Octocorallia re-examined. ZooKeys. 2013;272:29-59.

78. van Ofwegen LP, McFadden CS, Benayahu Y. Sinularia polydactyla Ehrenberg 1834 (Cnidaria, Octocorallia) re-examined with description of a new species. ZooKeys. 2016;581:71-126.

79. Pante E, Abdelkrim J, Viricel A, Gey D, France SC, Boisselier MC, Samadi S. Use of RAD sequencing for delimiting species. Heredity. 2015;114:450.

80. Paz-García DA, Hellberg ME, García-de-León FJ, Balart EF. Switch between morphospecies of Pocillopora corals. Am Nat. 2015;186:434-40.

81. Prada C, DeBiasse MB, Neigel JE, Yednock B, Stake JL, Forsman ZH, Hellberg ME. Genetic species delineation among branching Caribbean Porites corals. Coral Reefs. 2014;33:1019-30.

82. Pritchard JK, Stephens M, Rosenberg NA, Donnelly P. Association mapping in structured populations. Am J Hum Genet. 2000;67:170-81.

83. Quattrini AM, Faircloth BC, Dueñas LF, Bridge TC, Brugler MR, CalixtoBotía IF, et al. Universal target-enrichment baits for anthozoan (Cnidaria) phylogenomics: new approaches to long-standing problems. Mol Ecol Resour. 2018;18:281-95.

84. R Core Team. R: A Language and Environment for Statistical Computing. R Foundation for Statistical Computing Vienna Austria. 2012 http://www.rproject.org/. Accessed 15 Jan 2019.

85. Radice VZ, Quattrini AM, Wareham VE, Edinger EN, Cordes EE. Vertical water mass structure in the North Atlantic influences the bathymetric distribution of species in the deep-sea coral genus Paramuricea. Deep Sea Res I. 2016; 116:253-63.

86. Rambaut A, Drummond AJ. Tracer v1.4; 2007. p. 2012.
87. Richards ZT, van Oppen MJH, Wallace CC, Willis BL, Miller DJ. Some rare indo-Pacific coral species are probable hybrids. PLoS One. 2008;3:e3240.

88. Richmond $\mathrm{RH}$, Hunter $\mathrm{CL}$. Reproduction and recruitment of corals: comparisons among the Caribbean the tropical Pacific and the Red Sea. Mar Ecol Prog Ser. 1990;60:185-203.

89. Rheindt FE, Edwards SV. Genetic introgression: an integral but neglected component of speciation in birds. Auk. 2011;128:620-32.

90. Rogers AR, Bohlender RJ. Bias in estimators of archaic admixture. Theoretical Pop Biol. 2015;100:63-78

91. Rosenberg NA. DISTRUCT: a program for the graphical display of population structure. Mol Ecol Notes. 2004;4:137-8.

92. Rowley SJ, Pochon X, Watling L. Environmental influences on the indoPacific octocoral Isis hippuris Linnaeus 1758 Alcyonacea: Isididae: genetic fixation or phenotypic plasticity? PeerJ. 2015;3:e1128.

93. Sánchez JA, Aguilar C, Dorado D, Manrique N. Phenotypic plasticity and morphological integration in a marine modular invertebrate. BMC Evol Biol. 2007;7:122.

94. Schloss PD, Westcott SL, Ryabin T, Hall JR, Hartmann M, Hollister EB, Sahl JW. Introducing mothur: open-source platform-independent community-supported software for describing and comparing microbial communities. Appl Environ Microbiol. 2009;75:7537-41.

95. Schmieder R, Edwards R. Fast identification and removal of sequence contamination from genomic and metagenomic datasets. PLoS One. 2011;6:e17288.

96. Shearer TL, Coffroth MA. Barcoding corals: limited by interspecific divergence not intraspecific variation. Mol Ecol Resour. 2008;8:247-55.

97. Shoham E, Benayahu Y. Higher species richness of octocorals in the upper mesophotic zone in Eilat Gulf of Aqaba compared to shallower reef zones. Coral Reefs. 2017;36:71-81.

98. Shoham E, Prohaska T, Barkai Z, Zitek A, Benayahu Y. Soft corals form aragoniteprecipitated columnar spiculite in mesophotic reefs. Sci Rep. 2019;9:1241.

99. Slattery M, Hines GA, Starmer J, Paul VJ. Chemical signals in gametogenesis spawning and larval settlement and defense of the soft coral Sinularia polydactyla. Coral Reefs. 1999;18:75-84.

100. Slattery M, Starmer J, Paul VJ. Temporal and spatial variation in defensive metabolites of the tropical soft corals Sinularia maxima and S. polydactyla. Mar Biol. 2001;138:1183-93.

101. Slattery M, Kamel HN, Ankisetty S, Gochfield DJ, Hoover CA, Thacker RW. Hybrid vigor in a tropical Pacific soft-coral community. Ecol Monogr. 2008; 78:423-43.

102. Stamatakis A. RAxML-VI-HPC: maximum likelihood-based phylogenetic analyses with thousands of taxa and mixed models. Bioinformatics. 2006;22:2688-90.

103. Sukumaran J, Knowles LL. Multispecies coalescent delimits structure not species. PNAS. 2017:114:1607-12.

104. Titus BM, Daly M. Reduced representation sequencing for symbiotic anthozoans: are reference genomes necessary to eliminate endosymbiont contamination and make robust phylogeographic inference? bioRxiv. 2018; https://doi.org/10.1101/440289.

105. Tursch B, Tursch A. The soft coral community on a sheltered reef quadrat at Laing Island Papua New Guinea. Mar Biol. 1982;68:321-32.

106. Van Alstyne KL, Wylie CR, Paul VJ. Antipredator defenses in tropical Pacific soft corals Coelenterata: Alcyonacea II. The relative importance of chemical and structural defenses in three species of Sinularia. J Mar Biol Ecol. 1994;178:17-34.

107. van Oppen MV, Willis BL, Vugt HV, Miller DJ. Examination of species boundaries in the Acropora cervicornis group (Scleractinia, Cnidaria) using nuclear DNA sequence analyses. Mol Ecol. 2000;9:1363-73.

108. van Oppen MJ, McDonald BJ, Willis B, Miller DJ. The evolutionary history of the coral genus Acropora (Scleractinia, Cnidaria) based on a mitochondrial and a nuclear marker: reticulation incomplete lineage sorting or morphological convergence? Mol Biol Evol. 2001;18:1315-29.

109. van Oppen MJ, Willis BL, Van Rheede T, Miller DJ. Spawning times reproductive compatibilities and genetic structuring in the Acropora aspera group: evidence for natural hybridization and semi-permeable species boundaries in corals. Mol Ecol. 2002;11:1363-76.

110. Vargas S, Breedy O, Siles F, Guzman HM. How many kinds of sclerite? Towards a morphometric classification of gorgoniid microskeletal components. Micron. 2010;41:158-64.

111. Verseveldt J. A revision of the genus Sinularia May Octocorallia Alcyonacea. Zoologische Verhandelingen. 1980;179:1-128.

112. Vollmer SV, Palumbi SR. Hybridization and the evolution of reef coral diversity. Science. 2002;296:2023-5 
113. Willis BL, Babcock RC, Harrison PL, Wallace CC. Experimental hybridization and breeding incompatibilities within the mating systems of mass spawning reef corals. Coral Reefs. 1997;16:S53-65.

114. Willis BL, van Oppen MJ, Miller DJ, Vollmer SV, Ayre DJ. The role of hybridization in the evolution of reef corals. Annu Rev Ecol Evol Syst. 2006; 37:489-517.

115. WoRMS Editorial Board. World Register of Marine Species; 2018 http:// www.marinespecies.org at VLIZ. Accessed 19 Dec 2018. https://doi.org/10. 14284/170

116. Wylie CR, Paul VJ. Chemical defenses in three species of Sinularia Coelenterata Alcyonacea: effects against generalist predators and the butterflyfish Chaetodon unimaculatus Bloch. J Mar Biol Ecol. 1989;129:141-60.

117. Zwickl DJ. GARLl: genetic algorithm for rapid likelihood inference. 2006. http://www.bio.utexas.edu/faculty/antisense/garli/Garli.html. Accessed 15 Jan 2019.

Ready to submit your research? Choose BMC and benefit from:

- fast, convenient online submission

- thorough peer review by experienced researchers in your field

- rapid publication on acceptance

- support for research data, including large and complex data types

- gold Open Access which fosters wider collaboration and increased citations

- maximum visibility for your research: over $100 \mathrm{M}$ website views per year

At BMC, research is always in progress.

Learn more biomedcentral.com/submissions 